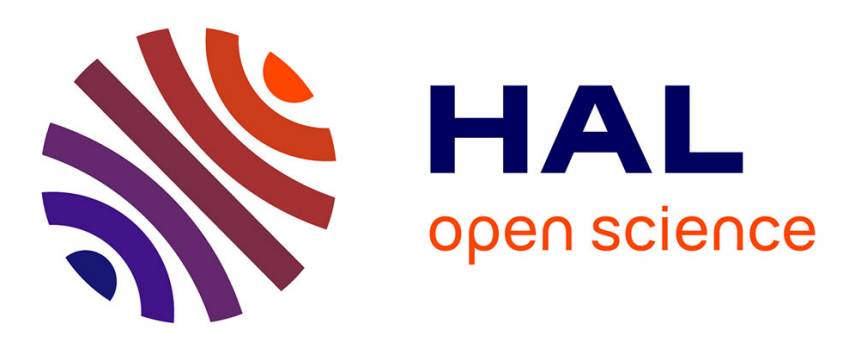

\title{
A degenerate parabolic-hyperbolic Cauchy problem with a stochastic force
}

Caroline Bauzet, Guy Vallet, Petra Wittbold

\section{To cite this version:}

Caroline Bauzet, Guy Vallet, Petra Wittbold. A degenerate parabolic-hyperbolic Cauchy problem with a stochastic force. Journal of Hyperbolic Differential Equations, 2015, 12 (03), pp.501-533. 10.1142/S0219891615500150 . hal-01003069

\section{HAL Id: hal-01003069 \\ https://hal.science/hal-01003069}

Submitted on 10 Jun 2014

HAL is a multi-disciplinary open access archive for the deposit and dissemination of scientific research documents, whether they are published or not. The documents may come from teaching and research institutions in France or abroad, or from public or private research centers.
L'archive ouverte pluridisciplinaire HAL, est destinée au dépôt et à la diffusion de documents scientifiques de niveau recherche, publiés ou non, émanant des établissements d'enseignement et de recherche français ou étrangers, des laboratoires publics ou privés. 


\title{
A degenerate parabolic-hyperbolic Cauchy problem with a stochastic force
}

\section{Caroline BAUZET* ${ }^{*}$ Guy VALLET ${ }^{\dagger} \quad$ Petra WITTBOLD ${ }^{\ddagger}$}

August 10, 2013

\begin{abstract}
In this paper we are interested in the Cauchy problem for a nonlinear degenerate parabolic-hyperbolic problem with multiplicative stochastic forcing. Using an adapted entropy formulation a result of existence and uniqueness of a solution is proved.

Keywords: Stochastic PDE ; degenerate parabolic-hyperbolic equation ; Cauchy problem ; multiplicative stochastic perturbation ; Carrillo-Kruzhkov's entropy.

MSC code: $35 \mathrm{~K} 65 ; 60 \mathrm{H} 15 ; 35 \mathrm{~L} 65$.
\end{abstract}

\section{Introduction}

In this paper, we are interested in the formal multi-dimensional $(d \geq 1)$ stochastic nonlinear degenerate parabolic problem of type:

$(P):\left\{\begin{array}{l}d u-\Delta \phi(u) d t-\operatorname{div}(\vec{f}(u)) d t=g(x, u) d t+h(x, u) d w \quad \text { in } \quad \Omega \times Q, \\ u(0, \cdot)=u_{0} \text { in } \mathbb{R}^{d}\end{array}\right.$

where, in the sequel we assume that $T$ is a positive number, $Q=] 0, T\left[\times \mathbb{R}^{d}\right.$ and that $W=\left\{w_{t}, \mathcal{F}_{t} ; 0 \leq t \leq T\right\}$ denotes a standard adapted one-dimensional continuous Brownian motion, defined on the classical Wiener space $(\Omega, \mathcal{F}, P)$. These assumptions on $W$ are made for convenience.

Let us assume that

$\mathrm{H}_{1}: \phi: \mathbb{R} \rightarrow \mathbb{R}$ is a Lipschitz-continuous function and $\phi(0)=0$.

*Institut de Mathmatiques de Marseille (I2M), Technopôle Chteau Gombert 39 rue F. Joliot Curie, 13453 Marseille Cedex 13 France

caroline.bauzet@univ-amu.fr

${ }^{\dagger}$ LMAP UMR - CNRS 5142, IPRA BP 1155, 64013 Pau Cedex France guy.vallet@univ-pau.fr

${ }^{\ddagger}$ Fakultät für Mathematik, Universitätsstr. 2, 45141 Essen Germany petra.wittbold@uni-due.de 
$\mathrm{H}_{2}: \vec{f}: \mathbb{R} \rightarrow \mathbb{R}^{d}$ is a Lipschitz-continuous function and $\vec{f}(0)=\overrightarrow{0}$.

$\mathrm{H}_{3}: g, h: \mathbb{R}^{d} \times \mathbb{R} \rightarrow \mathbb{R}$ are Carathéodory functions, Lipschitz-continuous with respect to the $\mathbb{R}$-variable, uniformly in the space variable, with $g(\cdot, 0), h(\cdot, 0)$ in $L^{2}\left(\mathbb{R}^{d}\right)$.

$\mathrm{H}_{4}: u_{0} \in L^{2}\left(\mathbb{R}^{d}\right)$.

$\mathrm{H}_{5}$ : for technical reasons we assume one of the following situations:

- Situation 1: For any $(x, u), h(x, u)=h(u)$;

- Situation $2^{1}: \phi=0$ or linear,

$$
|h(x, u)-h(y, v)| \leq c(h)\left(|u-v|+\omega_{h}(\|x-y\|)\right)
$$

for any $(x, u),(y, v)$, where $\omega_{h}$ is a modulus of continuity satisfying: there exists $\theta \in(0,1)$ such that $\frac{\omega_{h}(r)^{1+\theta}}{r} \rightarrow_{r \rightarrow 0} 0$

(this is the case for example if $\omega_{h}(r)=C|r|^{\beta}$ for a given $\beta>1 / 2$ by setting $1>\theta>(1-\beta) / \beta)$;

- Situation 3: assumptions concerning $h$ are the same as in the above case;

if $\phi$ is not linear, we assume that $t \mapsto \sqrt{\phi^{\prime}(t)}$ has a modulus of continuity $\omega_{\phi}$ such that $\frac{\omega_{\phi}\left[\omega_{h}(r)^{1+\theta}\right]}{r} \rightarrow_{r \rightarrow 0} 0$

(this is the case for example if $\left.\omega_{\phi}(r)=C|r|\right)^{2}$.

It is well-known, since J. Carrillo [3] in the deterministic case, that one needs an entropy formulation to prove that such degenerate parabolic problems are well-posed. Our aim is to adapt this formulation to the context of a stochastic problem. Since the "natural" framework of the above-cited author is $L^{1}\left(\mathbb{R}^{d}\right)$ and the "natural" framework of our SPDE is $L^{2}\left(\mathbb{R}^{d}\right)$, we had to revisit it through the ideas of G.-Q. Chen K.-H. Karlsen [2] and B. Andreianov and M. Maliki [4].

Concerning stochastic conservation laws in the literature, one can find some recent works. Let us cite without exhaustivity, for additive noises: W. E, K. Khanin and Y. Sinai [5] concerning the 1-D stochastic Burgers equation related to Hamilton-Jacobi equations; J.H. Kim [6], also in 1-D, for more general fluxes in the context of Kruzhkov's entropies; G. Vallet and P. Wittbold [7] where the authors considered a Dirichlet multidimensional problem in a bounded domain. There, semi-Kruzhkov entropies were considered in an entropy formulation "à la Carrillo" for the traces.

Concerning multiplicative noises, a first partial study was proposed by J. Feng and D. Nualart [8]. We mean partial since, based on Kruzhkov's techniques, the authors prove a result of uniqueness of the entropy solution for the Cauchy

\footnotetext{
${ }^{1}$ This situation generalizes [1].

${ }^{2}$ Such kind of assumption was made in [2].
} 
problem in $\mathbb{R}^{d}$ modulo the existence of what they have called a "strong-entropy" solution $^{3}$ and the existence of such a solution in $\mathbb{R}$. This study has been revisited by G.-Q. Chen, Q. Ding and K. H. Karlsen [9] where they proved the existence of a strong-entropy solution in the multidimensional case by using BV information on the initial condition.

The first general result of existence and uniqueness has been proposed by A. Debussche and J. Vovelle [10]. The problem is posed in a torus and the technique is based on the kinetic formulations associated to the equation. C. Bauzet, G. Vallet and P. Wittbold proposed in [1] a similar result by using Feng and Nualart's entropy formulation for the Cauchy problem in $\mathbb{R}^{d}$ in the framework of the Young measure theory. The same authors gave a similar result for the Dirichlet problem in [11].

To our knowledge, the only actual result concerning the case of a strongly degenerate parabolic-hyperbolic stochastic is a preprint of A. Debussche, M. Hofmanova and J. Vovelle extending the kinetic formulation in a torus of [10]. In this present paper, we propose to extend the previous paper [1] to the context of a degenerate parabolic-hyperbolic problem in the spirit of J. Carrillo's work [3] and revisited by G.-Q. Chen and K.-H. Karlsen [2]. Again, the existence of a solution is proved by using a vanishing viscosity method based on the compactness proposed by the theory of Young measures. The uniqueness of the solution is obtained via Kruzhkov's doubling variable method.

The paper is organized as follows. After this introductory part where we present some notations, we will present the entropy formulation, the definition of a solution and state the main result: the existence and uniqueness of the solution and some stability inequalities. Section 3 is devoted to the technical part of the paper where we show the existence of a solution and the uniqueness is presented in Section 4; followed by the last one containing technical lemmata.

Let us now introduce some notations and make precise the functional setting.

In the sequel we denote by $H^{1}\left(\mathbb{R}^{d}\right)$ the usual Sobolev space.

We recall that $H^{1}\left(\mathbb{R}^{d}\right)$ is also the closure of $\mathcal{D}\left(\mathbb{R}^{d}\right)$, the space of $C^{\infty}\left(\mathbb{R}^{d}\right)$ functions with compact support in $\mathbb{R}^{d}$. We denote by $H^{-1}\left(\mathbb{R}^{d}\right)$ the dual space of $H^{1}\left(\mathbb{R}^{d}\right)$ which is also the space of derivatives of order less than one of elements of $L^{2}\left(\mathbb{R}^{d}\right)$ in the common Gelfand-Lions identification $H^{1}\left(\mathbb{R}^{d}\right) \hookrightarrow L^{2}\left(\mathbb{R}^{d}\right) \equiv L^{2}\left(\mathbb{R}^{d}\right)^{\prime} \hookrightarrow H^{1}\left(\mathbb{R}^{d}\right)^{\prime}$.

For any positive $M$, denote by $\left.Q_{M}=\right] 0, T[\times B(0, M)$ where $B(0, M)$ is the bounded open ball in $\mathbb{R}^{d}$ of radius $M$.

In general, if $G \subset \mathbb{R}^{k}, \mathcal{D}(G)$ denotes the restriction to $G$ of $\mathcal{D}\left(\mathbb{R}^{k}\right)$ functions $u$ such that support $(u) \cap G$ is compact. Then, $\mathcal{D}^{+}(G)$ will denote the subset of non-negative elements of $\mathcal{D}(G)$.

\footnotetext{
${ }^{3}$ Here we don't mean pathwise, nor martingale solutions.
} 
For a given separable Banach space $X$ we denote by $N_{w}^{2}(0, T, X)$ the space of the predictable $X$-valued processes (cf. [12] p.94 or [13] p.28 for example). This space is the space $L^{2}(] 0, T[\times \Omega, X)$ for the product measure $d t \otimes d P$ on $\mathcal{P}_{T}$, the predictable $\sigma$-field (i.e. the $\sigma$-field generated by the sets $\{0\} \times \mathcal{F}_{0}$ and the rectangles $] s, t] \times A$ for any $\left.A \in \mathcal{F}_{s}\right)$, with the $L^{2}(] 0, T[\times \Omega, X)$ norm.

If $X=L^{2}\left(\mathbb{R}^{d}\right)$, one gets that $N_{w}^{2}\left(0, T, L^{2}\left(\mathbb{R}^{d}\right)\right) \subset L^{2}(Q \times \Omega)$.

We denote by $\mathcal{E}$ the set of non-negative even convex function in $C^{2,1}(\mathbb{R})$ approximating the absolute-value function, such that $\eta(0)=0$ and that there exists $\tau>0$ such that $\eta^{\prime}(x)=1$ (resp. -1 ) if $x>\tau$ (resp. $x<-\tau$ ). Then, $\eta^{\prime \prime}$ has a compact support in $[-\tau, \tau]$ and $\eta$ and $\eta^{\prime}$ are Lipschitz-continuous functions. A typical element of $\mathcal{E}$ is the function denoted by $\eta_{\tau}$ such that $\eta_{\tau}^{\prime}(r)=\frac{1+\sin \left(\frac{\pi}{2 \tau}(2 r-\tau)\right)}{2}$ if $0 \leq r \leq \tau$ and $\eta_{\tau}^{\prime}(r)=1$ if $r>\tau$.

For convenience, denote by $\operatorname{sgn}_{0}(x)=\frac{x}{|x|}$ if $x \neq 0$ and 0 otherwise;

$F(a, b)=\operatorname{sgn}_{0}(a-b)[\vec{f}(a)-\vec{f}(b)]$ and $F^{\eta}(a, b)=\int_{b}^{a} \eta^{\prime}(\sigma-b) \vec{f}^{\prime}(\sigma) d \sigma$.

Note, in particular, that $F$ and $F^{\eta}$ are Lipschitz-continuous functions.

Denote also: $\phi^{\eta}(a, b)=\int_{b}^{a} \eta^{\prime}(\sigma-b) \phi^{\prime}(\sigma) d \sigma$ and $G(x)=\int_{0}^{x} \sqrt{\phi^{\prime}(s)} d s$.

\section{Towards an entropy formulation and defini- tion of a solution}

Following the method proposed in G. Vallet $[14]^{1}$, for any $\epsilon>0$, there exists a unique solution $u$ in $N_{w}^{2}\left(0, T, H^{1}\left(\mathbb{R}^{d}\right)\right)$ with $\partial_{t}\left(u-\int_{0}^{t} h(x, u) d w\right)$ in $L^{2}(\Omega \times$ $\left.(0, T), H^{-1}\left(\mathbb{R}^{d}\right)\right)$, to Problem:

$\left(P_{\epsilon}\right):\left\{\begin{array}{l}\partial_{t}\left[u-\int_{0}^{t} h(x, u) d w\right]-\epsilon \Delta u-\Delta \phi(u)-\operatorname{div} \vec{f}(u)=g(x, u) \quad \text { in } \quad \Omega \times Q \\ u(0, \cdot)=u_{0} \text { in } \mathbb{R}^{d}\end{array}\right.$

Note that one has $u \in L^{2}\left(\Omega, C\left([0, T], L^{2}\left(\mathbb{R}^{d}\right)\right)\right.$.

Then, a slight modification of the Itô's formula proposed in D. Fellah and E. Pardoux [15], for any $\varphi \in D^{+}\left(\left[0, T\left[\times \mathbb{R}^{d}\right)\right.\right.$, any reals $v, k, \eta \in \mathcal{E}$ and $H(v, k)=$ $\eta(v-k)$, yields (denote by $\left.Q_{t}=(0, t) \times \mathbb{R}^{d}\right)$

$$
\begin{aligned}
& \int_{\mathbb{R}^{d}} H(u(t), k) \varphi(t) d x-\int_{\mathbb{R}^{d}} H\left(u_{0}, k\right) \varphi(0) d x+\epsilon \int_{Q_{t}} \nabla u \nabla\left[\eta^{\prime}(u-k) \varphi\right] d x d s \\
& +\int_{Q_{t}} \nabla \phi(u) \nabla\left[\eta^{\prime}(u-k) \varphi\right] d x d s+\int_{Q_{t}} \vec{f}(u) \nabla\left[\eta^{\prime}(u-k) \varphi\right] d x d s
\end{aligned}
$$

\footnotetext{
${ }^{1}$ To adapt the proof of the main result of this paper, one just needs to consider the resolvant $(I-\Delta)^{-1}$ instead of $(-\Delta)^{-1}$.
} 


$$
\begin{aligned}
= & \int_{Q_{t}} H(u, k) \partial_{t} \varphi d x d s+\int_{Q_{t}} \eta^{\prime}(u-k) \varphi h(x, u) d w(s) d x \\
& +\frac{1}{2} \int_{Q_{t}} \eta^{\prime \prime}(u-k)[h(x, u)]^{2} \varphi d x d s+\int_{Q_{t}} \eta^{\prime}(u-k) g(x, u) \varphi d x d s .
\end{aligned}
$$

Then, since

$$
\int_{Q_{t}} \eta^{\prime \prime}(u-k) \vec{f}(u) \nabla u \varphi d x d s+\int_{Q_{t}} \eta^{\prime}(u-k) \vec{f}(u) \nabla \varphi d x d s=\int_{Q_{t}} F^{\eta}(u, k) \nabla \varphi d x d s
$$

the following equality holds:

$$
\begin{aligned}
& \int_{\mathbb{R}^{d}} H(u(t), k) \varphi(t) d x+\epsilon \int_{Q_{t}} \eta^{\prime \prime}(u-k)|\nabla u|^{2} \varphi d x d s+\int_{Q_{t}} \eta^{\prime \prime}(u-k) \phi^{\prime}(u)|\nabla u|^{2} \varphi d x d s \\
& =-\epsilon \int_{Q_{t}} \eta^{\prime}(u-k) \nabla u \nabla \varphi d x d s+\int_{\mathbb{R}^{d}} H\left(u_{0}, k\right) \varphi(0) d x \\
& \quad+\int_{Q_{t}}\left(H(u, k) \partial_{t} \varphi-\eta^{\prime}(u-k) \nabla \phi(u) \nabla \varphi-F^{\eta}(u, k) \nabla \varphi\right) d x d s \\
& +\int_{Q_{t}} \eta^{\prime}(u-k) \varphi h(x, u) d w(s) d x+\int_{Q_{t}}\left[\eta^{\prime}(u-k) g(x, u)+\frac{1}{2} \eta^{\prime \prime}(u-k)[h(x, u)]^{2}\right] \varphi d x d s .
\end{aligned}
$$

Since $\phi^{\eta}(x, k)=\int_{k}^{x} \eta^{\prime}(\sigma-k) \phi^{\prime}(\sigma) d \sigma$ and $G(x)=\int_{0}^{x} \sqrt{\phi^{\prime}(s)} d s$, one gets that

$$
\begin{aligned}
& \int_{\mathbb{R}^{d}} H(u(t), k) \varphi(t) d x+\epsilon \int_{Q_{t}} \eta^{\prime \prime}(u-k)|\nabla u|^{2} \varphi d x d s+\int_{Q_{t}} \eta^{\prime \prime}(u-k)|\nabla G(u)|^{2} \varphi d x d s \\
& =-\epsilon \int_{Q_{t}} \eta^{\prime}(u-k) \nabla u \nabla \varphi d x d s+\int_{\mathbb{R}^{d}} H\left(u_{0}, k\right) \varphi(0) d x \\
& \quad+\int_{Q_{t}}\left(H(u, k) \partial_{t} \varphi+\phi^{\eta}(u, k) \Delta \varphi-F^{\eta}(u, k) \nabla \varphi\right) d x d s \\
& +\int_{Q_{t}} \eta^{\prime}(u-k) \varphi h(x, u) d w(s) d x+\int_{Q_{t}}\left[\eta^{\prime}(u-k) g(x, u)+\frac{1}{2} \eta^{\prime \prime}(u-k)[h(x, u)]^{2}\right] \varphi d x d s .
\end{aligned}
$$

Note that the second integral on the left hand side is non-negative.

Moreover, one might expect that the first integral term on the right hand side of the equation tends to 0 as $\epsilon$ tends to 0 .

Therefore, if we can show that the solutions of $\left(P_{\epsilon}\right)$ converge in an appropriate sense to a function $u$ as $\epsilon$ tends to 0 , the limit function will satisfy the entropy inequality (1) where $\epsilon=0$ and the equality sign is replaced by an inequality.

So we propose 
Definition 1 A solution to Problem $(P)$ is any $u \in N_{w}^{2}\left(0, T, L^{2}\left(\mathbb{R}^{d}\right)\right) \cap L^{\infty}\left(0, T, L^{2}(\Omega \times\right.$ $\left.\mathbb{R}^{d}\right)$ ) such that $G(u) \in L^{2}\left((0, T) \times \Omega, H^{1}\left(\mathbb{R}^{d}\right)\right)$ and satisfying, a.s. the entropy formulation: $\forall k \in \mathbb{R}, \forall \varphi \in D^{+}\left(\left[0, T\left[\times \mathbb{R}^{d}\right), \forall \eta \in \mathcal{E}\right.\right.$,

$$
\begin{aligned}
& \int_{Q}\left(H(u, k) \partial_{t} \varphi+\phi^{\eta}(u, k) \Delta \varphi-F^{\eta}(u, k) \nabla \varphi+\eta^{\prime}(u-k) g(x, u) \varphi\right) d x d s \\
& +\int_{Q} \eta^{\prime}(u-k) \varphi h(x, u) d w(s) d x+\frac{1}{2} \int_{Q} \eta^{\prime \prime}(u-k)[h(x, u)]^{2} \varphi d x d s \\
\geq & \int_{Q} \eta^{\prime \prime}(u-k)|\nabla G(u)|^{2} \varphi d x d s-\int_{\mathbb{R}^{d}} H\left(u_{0}, k\right) \varphi(0) d x .
\end{aligned}
$$

Let us first make some remarks on the definition.

Remark 1 1. Note that if $G(u) \in L^{2}\left((0, T) \times \Omega, H^{1}\left(\mathbb{R}^{d}\right)\right)$, then $\phi(u) \in$ $L^{2}\left((0, T) \times \Omega, H^{1}\left(\mathbb{R}^{d}\right)\right)$ and, thanks to Lemma 3 (see Section 5), the entropy inequality is equivalent to

$$
\begin{aligned}
& \int_{Q}\left(H(u, k) \partial_{t} \varphi+\phi^{\eta}(u, k) \Delta \varphi-F^{\eta}(u, k) \nabla \varphi+\eta^{\prime}(u-k) g(x, u) \varphi\right) d x d s \\
+ & \int_{Q} \eta^{\prime}(u-k) \varphi h(x, u) d w(s) d x+\frac{1}{2} \int_{Q} \eta^{\prime \prime}(u-k)[h(x, u)]^{2} \varphi d x d s . \\
\geq & \int_{Q}\left|\nabla \int_{0}^{u} \sqrt{\eta^{\prime \prime}(\sigma-k)} G^{\prime}(\sigma) d \sigma\right|^{2} \varphi d x d s-\int_{\mathbb{R}^{d}} H\left(u_{0}, k\right) \varphi(0) d x
\end{aligned}
$$

2. Following Remark 2.6 in [1], one has that a solution in the sense of the above definition is also, a.s., a weak solution of $(P)$.

3. Following now Remark 2.7 of the same paper, one gets that a solution $u$ in the sense of the above definition satisfies $\underset{t \rightarrow 0^{+}}{\text {ess }} \lim \int_{K}\left|u-u_{0}\right| d x=0$ for any compact $K$ of $\mathbb{R}^{d}$, but also, ess $\lim _{t \rightarrow 0^{+}} E \int_{\mathbb{R}^{d}}\left|u-u_{0}\right| \varphi(x) d x=0$ for any $\varphi \in L^{2}\left(\mathbb{R}^{d}\right)$.

Let us also remark that any solution $u$ belongs to $L^{2}\left[(0, T), L^{2}\left(\Omega \times \mathbb{R}^{d}\right)\right]$, and it is the same for $u-\int_{0}^{t} h(x, u) d w(s)$ thanks to the properties of the Itô integral. As u is also a weak solution of $(P), \partial_{t}\left[u-\int_{0}^{t} h(x, u) d w(s)\right]$ belongs to $L^{2}\left[(0, T), L^{2}\left(\Omega, H^{-1}\left(\mathbb{R}^{d}\right)\right)\right]$.

Thus, $u \in C\left([0, T], L^{2}\left(\Omega, H^{-1}\left(\mathbb{R}^{d}\right)\right)\right)$. Since by definition $u$ belongs to $L^{\infty}\left(0, T, L^{2}\left(\Omega \times \mathbb{R}^{d}\right)\right)$, Lemma 1.4 p.263 of [16] yields: $u$ is weakly continuous in time with values in $L^{2}\left(\Omega \times \mathbb{R}^{d}\right)$.

Let us now present the main result of the paper. 


\section{Theorem 1}

Under the assumptions $H_{1}$ to $H_{4}$, there exists a unique solution in the sense of Definition 1.

Considering two initial conditions $u_{0,2}, u_{0,1}$, the corresponding solutions $u_{2}, u_{1}$ and the weight $\alpha(x)=\min \left(1, \frac{R^{a}}{|x|^{a}}\right)$ where $R>1$ and $a>d / 2$, there exists $c>0$ such that for any positive $t$ :

$$
E \int_{\mathbb{R}^{d}}\left|u_{2}(t, x)-u_{1}(t, x)\right| \alpha(x) d x \leq e^{c t} \int_{\mathbb{R}^{d}}\left|u_{0,2}(x)-u_{0,1}(x)\right| \alpha(x) d x .
$$

Moreover, if the initial conditions and $g(\cdot, 0)$ are also elements of $L^{1}\left(\mathbb{R}^{d}\right)$ and $h(\cdot, 0)=0$, then the solutions are in $L^{\infty}\left(0, T, L^{1}\left(\Omega \times \mathbb{R}^{d}\right)\right)$ and one has for any $t,\left\|\left(u_{2}-u_{1}\right)(t)\right\|_{L^{1}\left(\Omega \times \mathbb{R}^{d}\right)} \leq e^{c t}\left\|u_{0,2}-u_{0,1}\right\|_{L^{1}\left(\mathbb{R}^{d}\right)}$.

\section{Existence of a solution}

Let us denote in the sequel $u^{\epsilon}$ the solution of Problem $\left(P_{\epsilon}\right)$ with initial condition $u_{0}^{\epsilon} \in D\left(\mathbb{R}^{d}\right)$ that converges to a given $u_{0,2}$ in $L^{2}\left(\mathbb{R}^{d}\right)$ and consider $u^{\delta}$ a solution of Problem $\left(P_{\delta}\right)$ with initial condition $u_{0}^{\delta} \in D\left(\mathbb{R}^{d}\right)$ that converges to a given $u_{0,1}$ in $L^{2}\left(\mathbb{R}^{d}\right)$.

Based on the Kruzhkov's doubling variables method, our aim in this section is formally to pass to the limit when $\epsilon$ and $\delta$ go to 0 in a Kato's inequality. The compactness we use is the one given by the theory of Young measures and the classical uniqueness method for entropy solutions ensures the uniqueness of the limit point of the sequence of viscous approximation. This then yields the convergence of the whole sequence to an entropy solution in the sense of Definition 1.

To prove such Kato's inequality, [1] used that $\Delta u^{\delta} \in L^{2}(\Omega \times Q)$. In the present case, such a regularity is not possible to obtain and one needs to regularize $u^{\delta}$ by convolution.

Then, for a given mollifier-sequence $\rho_{\theta}$ in $\mathbb{R}^{d}$, using in the equation satisfied by $u^{\delta}$ the test function $\varphi * \rho_{\theta}$ for any $\varphi \in \mathcal{D}\left(\mathbb{R}^{d+1}\right)$, one gets that $u^{\delta} * \rho_{\theta}$ is a solution to the stochastic problem ${ }^{1}: u^{\delta} * \rho_{\theta}(t=0)=u_{0}^{\delta} * \rho_{\theta}$ and

$$
\begin{array}{r}
\partial_{t}\left[u^{\delta} * \rho_{\theta}-\int_{0}^{t} h\left(x, u^{\delta}\right) * \rho_{\theta} d w\right]-\left[\delta \Delta\left(u^{\delta} * \rho_{\theta}\right)+\Delta\left(\phi\left(u^{\delta}\right) * \rho_{\theta}\right)+\right. \\
=g\left(x, u^{\delta}\right) * \rho_{\theta}
\end{array}
$$

Note in particular that this problem is posed in $L^{2}\left(\mathbb{R}^{d}\right)$ and not anymore in $H^{-1}\left(\mathbb{R}^{d}\right)$.

Then, for any $\varphi \in D\left(\left[0, T\left[\times \mathbb{R}^{d}\right)\right.\right.$ (when needed in the sequel, one denotes by $K$ the support of $\varphi)$ any real $k$, the Itô formula applied to $H\left(u^{\delta} * \rho_{\theta}(t, x), k\right) \varphi(t, x)$

\footnotetext{
${ }^{1}$ One uses that $\rho_{\theta}$ is even and the properties of the Itô integral with continuous linear operators.
} 
where $\eta \in \mathcal{E}$ and $H(v, k)=\eta(v-k)$, yields a.e.

$$
\begin{aligned}
& H\left(u^{\delta} * \rho_{\theta}(t), k\right) \varphi(t)-H\left(u_{0}^{\delta} * \rho_{\theta}, k\right) \varphi(0)-\delta \int_{0}^{t} \Delta\left[u^{\delta} * \rho_{\theta}\right]\left[\eta^{\prime}\left(u^{\delta} * \rho_{\theta}-k\right) \varphi\right] d s \\
& -\int_{0}^{t} \Delta\left[\phi\left(u^{\delta}\right) * \rho_{\theta}\right]\left[\eta^{\prime}\left(u^{\delta} * \rho_{\theta}-k\right) \varphi\right] d s-\int_{0}^{t} \operatorname{div}\left[\vec{f}\left(u^{\delta}\right) * \rho_{\theta}\right]\left[\eta^{\prime}\left(u^{\delta} * \rho_{\theta}-k\right) \varphi\right] d s \\
= & \int_{0}^{t} H\left(u^{\delta} * \rho_{\theta}, k\right) \partial_{t} \varphi d s+\int_{0}^{t} \eta^{\prime}\left(u^{\delta} * \rho_{\theta}-k\right) \varphi\left[h\left(x, u^{\delta}\right) * \rho_{\theta}\right] d w(s) \\
& +\int_{0}^{t}\left[\eta^{\prime}\left(u^{\delta} * \rho_{\theta}-k\right) g\left(x, u^{\delta}\right) * \rho_{\theta}+\frac{1}{2} \eta^{\prime \prime}\left(u^{\delta} * \rho_{\theta}-k\right)\left[h\left(x, u^{\delta}\right) * \rho_{\theta}\right]^{2}\right] \varphi d s
\end{aligned}
$$

i.e., by integrating over $Q$

$$
\begin{aligned}
& \delta \int_{Q} \eta^{\prime \prime}\left(u^{\delta} * \rho_{\theta}-k\right)\left|\nabla\left[u^{\delta} * \rho_{\theta}\right]\right|^{2} \varphi d x d s+\delta \int_{Q} \eta^{\prime}\left(u^{\delta} * \rho_{\theta}-k\right) \nabla\left[u^{\delta} * \rho_{\theta}\right] \nabla \varphi d x d s \\
& +\int_{Q} \eta^{\prime \prime}\left(u^{\delta} * \rho_{\theta}-k\right) \nabla\left[\phi\left(u^{\delta}\right) * \rho_{\theta}\right] \nabla\left[u^{\delta} * \rho_{\theta}\right] \varphi d x d s \\
& +\int_{Q} \eta^{\prime}\left(u^{\delta} * \rho_{\theta}-k\right) \nabla\left[\phi\left(u^{\delta}\right) * \rho_{\theta}\right] \nabla \varphi d x d s \\
& +\int_{Q} \eta^{\prime \prime}\left(u^{\delta} * \rho_{\theta}-k\right)\left[\vec{f}\left(u^{\delta}\right) * \rho_{\theta}\right] \nabla\left[u^{\delta} * \rho_{\theta}\right] \varphi d x d s \\
& +\int_{Q} \eta^{\prime}\left(u^{\delta} * \rho_{\theta}-k\right)\left[\vec{f}\left(u^{\delta}\right) * \rho_{\theta}\right] \nabla \varphi d x d s \\
& =\int_{Q} H\left(u^{\delta} * \rho_{\theta}, k\right) \partial_{t} \varphi d x d s+\int_{Q} \eta^{\prime}\left(u^{\delta} * \rho_{\theta}-k\right) \varphi\left[h\left(x, u^{\delta}\right) * \rho_{\theta}\right] d w(s) d x \\
& +\int_{\mathbb{R}^{d}} H\left(u_{0}^{\delta} * \rho_{\theta}, k\right) \varphi(0) d x \\
& +\int_{Q}\left[\eta^{\prime}\left(u^{\delta} * \rho_{\theta}-k\right) g\left(x, u^{\delta}\right) * \rho_{\theta}+\frac{1}{2} \eta^{\prime \prime}\left(u^{\delta} * \rho_{\theta}-k\right)\left[h\left(x, u^{\delta}\right) * \rho_{\theta}\right]^{2}\right] \varphi d x d s .
\end{aligned}
$$

Or, if one agrees to denote, for any $v$ in $L^{2}\left(\mathbb{R}^{d}\right), v * \rho_{\theta}$ by $v_{\theta}$,

$$
\begin{aligned}
& \delta \int_{Q} \eta^{\prime \prime}\left(u_{\theta}^{\delta}-k\right)\left|\nabla u_{\theta}^{\delta}\right|^{2} \varphi d x d s+\int_{Q} \eta^{\prime \prime}\left(u_{\theta}^{\delta}-k\right) \nabla \phi\left(u^{\delta}\right)_{\theta} \nabla u_{\theta}^{\delta} \varphi d x d s \\
= & -\delta \int_{Q} \eta^{\prime}\left(u_{\theta}^{\delta}-k\right) \nabla u_{\theta}^{\delta} \nabla \varphi d x d s+\int_{\mathbb{R}^{d}} H\left(u_{0}^{\delta} * \rho_{\theta}, k\right) \varphi(0) d x \\
& +\int_{Q} H\left(u_{\theta}^{\delta}, k\right) \partial_{t} \varphi-\eta^{\prime}\left(u_{\theta}^{\delta}-k\right) \nabla \phi\left(u^{\delta}\right)_{\theta} \nabla \varphi-\eta^{\prime}\left(u_{\theta}^{\delta}-k\right)\left[\vec{f}\left(u^{\delta}\right)_{\theta}\right] \nabla \varphi d x d s \\
& -\int_{Q} \eta^{\prime \prime}\left(u_{\theta}^{\delta}-k\right)\left[\vec{f}\left(u^{\delta}\right)_{\theta}\right] \nabla u_{\theta}^{\delta} \varphi d x d s+\int_{Q} \eta^{\prime}\left(u_{\theta}^{\delta}-k\right) \varphi h\left(x, u^{\delta}\right)_{\theta} d w(s) d x \\
& +\int_{Q}\left[\eta^{\prime}\left(u_{\theta}^{\delta}-k\right) g\left(x, u^{\delta}\right)_{\theta}+\frac{1}{2} \eta^{\prime \prime}\left(u_{\theta}^{\delta}-k\right)\left[h\left(x, u^{\delta}\right)_{\theta}\right]^{2}\right] \varphi d x d s .
\end{aligned}
$$


In the sequel of this section, unless for the two integrals with $\epsilon$ as a factor term $^{2}$, we will present the proofs in such a way that it can also be done for a entropy solution $u$ (i.e. $\epsilon=0$ ). The main regularity difference between $u^{\epsilon}$ and $u$ is that $u^{\epsilon} \in H^{1}\left(\mathbb{R}^{d}\right)$ while $u \in L^{2}\left(\mathbb{R}^{d}\right)$ with $G(u) \in H^{1}\left(\mathbb{R}^{d}\right)$. So we need to use carefully a chain-rule; instead of the classical one, we will use a generalized chain-rule (see Lemma 3).

Let $\psi$ be an element of $D^{+}\left(\left[0, T\left[^{2} \times \mathbb{R}^{2 d}\right)\right.\right.$. The idea in the sequel is to replace $\psi(t, s, x, y)$ by $\varphi(t, x) \rho_{n}(t-s) \rho_{m}(x-y)$ for a given $\varphi \in D^{+}([0, T[\times \mathbb{R})$ and mollifier sequences $\rho_{n}$ in time with supp $\rho_{n} \subset\left[\frac{-2}{n}, 0\right]$ and $\rho_{m}$ in space with sufficiently large $n$ and $m$. Thus, multiplying (1) at time $t=T$ by $\rho_{l}\left[u^{\delta} *\right.$ $\left.\rho_{\theta}(s, y)-k\right]$ and integrating the result over $\mathbb{R} \times Q$ for the variables $k, s$ and $y$, yields

$$
\begin{aligned}
& \epsilon \int_{\mathbb{R} \times Q^{2}} \eta^{\prime \prime}\left(u^{\epsilon}-k\right)\left|\nabla u^{\epsilon}\right|^{2} \psi \rho_{l}\left[u_{\theta}^{\delta}(s, y)-k\right] d k d x d t d s d y \\
& +\int_{\mathbb{R} \times Q^{2}} \eta^{\prime \prime}\left(u^{\epsilon}-k\right)\left|\nabla G\left(u^{\epsilon}\right)\right|^{2} \psi \rho_{l}\left[u_{\theta}^{\delta}(s, y)-k\right] d k d x d t d s d y \\
= & -\epsilon \int_{\mathbb{R} \times Q^{2}} \eta^{\prime}\left(u^{\epsilon}-k\right) \nabla u^{\epsilon} \nabla_{x} \psi \rho_{l}\left[u_{\theta}^{\delta}(s, y)-k\right] d k d x d t d s d y \\
& +\int_{\mathbb{R} \times \mathbb{R}^{d} \times Q} H\left(u_{0}^{\epsilon}, k\right) \psi(0) \rho_{l}\left[u_{\theta}^{\delta}(s, y)-k\right] d k d x d s d y \\
& +\int_{\mathbb{R} \times Q^{2}}\left(H\left(u^{\epsilon}, k\right) \partial_{t} \psi+\phi^{\eta}\left(u^{\epsilon}, k\right) \Delta_{x} \psi-F^{\eta}\left(u^{\epsilon}, k\right) \nabla_{x} \psi+\eta^{\prime}\left(u^{\epsilon}-k\right) g\left(x, u^{\delta}\right)_{\theta} \varphi\right) \\
& +\int_{\mathbb{R} \times Q^{2}} \eta^{\prime}\left(u^{\epsilon}-k\right) \psi h\left(x, u^{\epsilon}\right) d w(t) \rho_{l}\left[u_{\theta}^{\delta}(s, y)-k\right] d k d x d s d y \\
& +\frac{1}{2} \int_{\mathbb{R} \times Q^{2}} \eta^{\prime \prime}\left(u^{\epsilon}-k\right)\left[h\left(x, u^{\epsilon}\right)\right]^{2} \psi \rho_{l}\left[u_{\theta}^{\delta}(s, y)-k\right] d k d t d x d s d x d t d s d y .
\end{aligned}
$$

Similarly, considering (2) and multiplying by $\rho_{l}\left[u^{\epsilon}(t, x)-k\right]$ and integrating with respect to $k, x$ and $t$,

$$
\begin{aligned}
& \delta \int_{\mathbb{R} \times Q^{2}} \eta^{\prime \prime}\left(u_{\theta}^{\delta}-k\right)\left|\nabla u_{\theta}^{\delta}\right|^{2} \psi \rho_{l}\left[u^{\epsilon}(t, x)-k\right] d y d s d k d x d t \\
& +\int_{\mathbb{R} \times Q^{2}} \eta^{\prime \prime}\left(u_{\theta}^{\delta}-k\right) \nabla \phi\left(u^{\delta}\right)_{\theta} \nabla u_{\theta}^{\delta} \rho_{l}\left[u^{\epsilon}(t, x)-k\right] \psi d y d s d k d x d t \\
= & -\delta \int_{\mathbb{R} \times Q^{2}} \eta^{\prime}\left(u_{\theta}^{\delta}-k\right) \nabla u_{\theta}^{\delta} \nabla_{y} \psi \rho_{l}\left[u^{\epsilon}(t, x)-k\right] d y d s d k d x d t \\
& +\int_{Q \times \mathbb{R} \times \mathbb{R}^{d}} H\left(u_{0, \theta}^{\delta}, k\right) \psi(0) \rho_{l}\left[u^{\epsilon}(t, x)-k\right] d y d k d x d t \\
& +\int_{\mathbb{R} \times Q^{2}}\left(H\left(u_{\theta}^{\delta}, k\right) \partial_{s} \psi-\eta^{\prime}\left(u_{\theta}^{\delta}-k\right) \nabla \phi\left(u^{\delta}\right)_{\theta} \nabla_{y} \psi-\eta^{\prime}\left(u_{\theta}^{\delta}-k\right)\left[\vec{f}\left(u^{\delta}\right)_{\theta}\right] \nabla_{y} \psi\right.
\end{aligned}
$$

\footnotetext{
${ }^{2}$ integrals that will disappear when $\epsilon$ will go to 0
} 


$$
\begin{array}{r}
\left.+\eta^{\prime}\left(u_{\theta}^{\delta}-k\right)\left[g\left(y, u^{\delta}\right)_{\theta}\right] \psi\right) \rho_{l}\left[u^{\epsilon}(t, x)-k\right] d y d s d k d x d t \\
-\int_{\mathbb{R} \times Q^{2}} \eta^{\prime \prime}\left(u_{\theta}^{\delta}-k\right)\left[\vec{f}\left(u^{\delta}\right)_{\theta}\right] \nabla u_{\theta}^{\delta} \psi \rho_{l}\left[u^{\epsilon}(t, x)-k\right] d y d s d k d x d t \\
+\int_{\mathbb{R} \times Q^{2}} \eta^{\prime}\left(u_{\theta}^{\delta}-k\right) \psi h\left(y, u^{\delta}\right)_{\theta} d w(s) \rho_{l}\left[u^{\epsilon}(t, x)-k\right] d y \\
+\frac{1}{2} \int_{\mathbb{R} \times Q^{2}} \eta^{\prime \prime}\left(u_{\theta}^{\delta}-k\right)\left[h\left(y, u^{\delta}\right)_{\theta}\right]^{2} \psi \rho_{l}\left[u^{\epsilon}(t, x)-k\right] d s d y d k d x d t
\end{array}
$$

Adding the two equations, by grouping similar terms together, we get:

$$
\begin{aligned}
& \epsilon \int_{\mathbb{R} \times Q^{2}} \eta^{\prime \prime}\left(u^{\epsilon}-k\right)\left|\nabla u^{\epsilon}\right|^{2} \psi \rho_{l}\left[u_{\theta}^{\delta}(s, y)-k\right] d x d t d k d y d s \\
& +\delta \int_{\mathbb{R} \times Q^{2}} \eta^{\prime \prime}\left(u_{\theta}^{\delta}-k\right)\left|\nabla u_{\theta}^{\delta}\right|^{2} \psi \rho_{l}\left[u^{\epsilon}(t, x)-k\right] d y d s d k d x d t \\
& +\int_{Q \times \mathbb{R} \times Q} \eta^{\prime \prime}\left(u^{\epsilon}-k\right)\left|\nabla G\left(u^{\epsilon}\right)\right|^{2} \psi \rho_{l}\left[u_{\theta}^{\delta}(s, y)-k\right] d x d t d k d y d s \\
& +\int_{Q \times \mathbb{R} \times Q} \eta^{\prime \prime}\left(u_{\theta}^{\delta}-k\right)\left[\nabla \phi\left(u^{\delta}\right)_{\theta} \nabla u_{\theta}^{\delta}\right] \psi \rho_{l}\left[u^{\epsilon}(t, x)-k\right] d y d s d k d x d t \\
& =-\epsilon \int_{\mathbb{R} \times Q^{2}} \eta^{\prime}\left(u^{\epsilon}-k\right) \nabla u^{\epsilon} \nabla_{x} \psi \rho_{l}\left[u_{\theta}^{\delta}(s, y)-k\right] d x d t d k d y d s \\
& -\delta \int_{\mathbb{R} \times Q^{2}} \eta^{\prime}\left(u_{\theta}^{\delta}-k\right) \nabla u_{\theta}^{\delta} \nabla_{y} \psi \rho_{l}\left[u^{\epsilon}(t, x)-k\right] d y d s d k d x d t \\
& +\int_{Q \times \mathbb{R} \times \mathbb{R}^{d}} H\left(u_{0}^{\epsilon}, k\right) \psi(t=0) \rho_{l}\left[u_{\theta}^{\delta}(s, y)-k\right] d x d k d y d s \\
& +\int_{Q \times \mathbb{R} \times \mathbb{R}^{d}} H\left(u_{0, \theta}^{\delta}, k\right) \psi(s=0) \rho_{l}\left[u^{\epsilon}(t, x)-k\right] d y d k d x d t \\
& +\int_{\mathbb{R} \times Q^{2}}\left(H\left(u^{\epsilon}, k\right) \partial_{t} \psi+\phi^{\eta}\left(u^{\epsilon}, k\right) \Delta_{x} \psi-F^{\eta}\left(u^{\epsilon}, k\right) \nabla_{x} \psi\right) \\
& \times \rho_{l}\left[u_{\theta}^{\delta}(s, y)-k\right] d x d t d k d y d s \\
& +\int_{\mathbb{R} \times Q^{2}}\left(H\left(u_{\theta}^{\delta}, k\right) \partial_{s} \psi-\eta^{\prime}\left(u_{\theta}^{\delta}-k\right) \nabla \phi\left(u^{\delta}\right)_{\theta} \nabla_{y} \psi-\eta^{\prime}\left(u_{\theta}^{\delta}-k\right)\left[\vec{f}\left(u^{\delta}\right)_{\theta} \nabla_{y} \psi\right]\right) \\
& \rho_{l}\left[u^{\epsilon}(t, x)-k\right] d y d s d k d x d t \\
& -\int_{\mathbb{R} \times Q^{2}} \eta^{\prime \prime}\left(u_{\theta}^{\delta}-k\right)\left[\vec{f}\left(u^{\delta}\right)_{\theta} \nabla u_{\theta}^{\delta}\right] \psi \rho_{l}\left[u^{\epsilon}(t, x)-k\right] d y d s d k d x d t \\
& +\frac{1}{2} \int_{\mathbb{R} \times Q^{2}} \eta^{\prime \prime}\left(u^{\epsilon}-k\right)\left[h\left(x, u^{\epsilon}\right)\right]^{2} \psi \rho_{l}\left[u_{\theta}^{\delta}(s, y)-k\right] d t d x d k d y d s \\
& +\frac{1}{2} \int_{\mathbb{R} \times Q^{2}} \eta^{\prime \prime}\left(u_{\theta}^{\delta}-k\right)\left[h\left(y, u^{\delta}\right)_{\theta}\right]^{2} \psi \rho_{l}\left[u^{\epsilon}(t, x)-k\right] d y d s d k d x d t \\
& +\int_{\mathbb{R} \times Q^{2}} \eta^{\prime}\left(u^{\epsilon}-k\right) \psi h\left(x, u^{\epsilon}\right) d w(t) \rho_{l}\left[u_{\theta}^{\delta}(s, y)-k\right] d x d k d y d s
\end{aligned}
$$




$$
\begin{aligned}
& +\int_{\mathbb{R} \times Q^{2}} \eta^{\prime}\left(u_{\theta}^{\delta}-k\right) \psi h\left(y, u^{\delta}\right)_{\theta} d w(s) \rho_{l}\left[u^{\epsilon}(t, x)-k\right] d y d k d x d t \\
& +\int_{\mathbb{R} \times Q^{2}} \eta^{\prime}\left(u^{\epsilon}-k\right) \psi g\left(x, u^{\epsilon}\right) d t \rho_{l}\left[u_{\theta}^{\delta}(s, y)-k\right] d x d k d y d s \\
& +\int_{\mathbb{R} \times Q^{2}} \eta^{\prime}\left(u_{\theta}^{\delta}-k\right) \psi g\left(y, u^{\delta}\right)_{\theta} d s \rho_{l}\left[u^{\epsilon}(t, x)-k\right] d y d k d x d t,
\end{aligned}
$$

i.e., $I_{1}+I_{2}=I_{3}+I_{4}+I_{5}+I_{6}+I_{7}+I_{8}$, where each $I_{j}$ denotes a sum of two corresponding integrals of the same type in the above equality.

Let us now study each of the terms $I_{1}, \cdots, I_{8}$ in detail. Our aim is to pass to the limit, successively with first $n$ to infinity, then $\theta$ to $0, l$ to infinity, then $\epsilon, \delta$ to 0 . Then, depending on the situation ( 1 to 3 ), we pass to the limit with respect to $\tau$ to 0 (i.e. with $\eta=\eta_{\tau}$ to the absolute-value function) and $m$ to infinity, in an appropriate order.

In the sequel, we adopt the following notation: $\lim _{a, b}$ means $\lim _{b} \lim _{a}$, also with limsup or liminf.

1) Since $\eta$ is a convex function,

$$
\begin{aligned}
I_{1}:= & \epsilon \int_{Q^{2} \times \mathbb{R}} \eta^{\prime \prime}\left(u^{\epsilon}-k\right)\left|\nabla u^{\epsilon}\right|^{2} \psi \rho_{l}\left[u_{\theta}^{\delta}(s, y)-k\right] d x d t d k d y d s \\
& +\delta \int_{Q^{2} \times \mathbb{R}} \eta^{\prime \prime}\left(u_{\theta}^{\delta}-k\right)\left|\nabla u_{\theta}^{\delta}\right|^{2} \psi \rho_{l}\left[u^{\epsilon}(t, x)-k\right] d y d s d k d x d t \\
\geq & 0,
\end{aligned}
$$

so, this term can be omitted in the sequel.

2) Remind that $G(x)=\int_{0}^{x} \sqrt{\phi^{\prime}(\sigma)} d \sigma$. Consider now

$$
\begin{aligned}
I_{2}:= & \int_{Q \times \mathbb{R} \times Q} \eta^{\prime \prime}\left(u^{\epsilon}-k\right)\left|\nabla G\left(u^{\epsilon}\right)\right|^{2} \psi \rho_{l}\left[u_{\theta}^{\delta}(s, y)-k\right] d x d t d k d y d s \\
& +\int_{Q \times \mathbb{R} \times Q} \eta^{\prime \prime}\left(u_{\theta}^{\delta}-k\right)\left[\nabla \phi\left(u^{\delta}\right)_{\theta} \nabla u_{\theta}^{\delta}\right] \psi \rho_{l}\left[u^{\epsilon}(t, x)-k\right] d y d s d k d x d t
\end{aligned}
$$

Then, replacing $\psi(t, s, x, y)$ by $\varphi(t, x) \rho_{n}(t-s) \rho_{m}(x-y)$, classical properties of Lebesgue's points and convolution yield

$$
\begin{aligned}
\lim _{n} E I_{2}= & E \int_{Q \times \mathbb{R} \times \mathbb{R}^{d}} \eta^{\prime \prime}\left(u^{\epsilon}-k\right)\left|\nabla G\left(u^{\epsilon}\right)\right|^{2} \varphi \rho_{m}(x-y) \rho_{l}\left[u_{\theta}^{\delta}(t, y)-k\right] d x d t d k d y \\
& +E \int_{Q \times \mathbb{R} \times \mathbb{R}^{d}} \eta^{\prime \prime}\left(u_{\theta}^{\delta}-k\right)\left[\nabla \phi\left(u^{\delta}\right)_{\theta} \nabla u_{\theta}^{\delta}\right] \varphi \rho_{m}(x-y) \rho_{l}\left[u^{\epsilon}(t, x)-k\right] d y d k d x d t
\end{aligned}
$$

Again, by properties of approximation by mollification, $G\left(u^{\epsilon}\right), G\left(u^{\delta}\right) \in L^{2}(\Omega \times$ $\left.(0, T) ; H^{1}\left(\mathbb{R}^{d}\right)\right)$ and since the nonlinear functions are bounded, one has

$$
\lim _{n, \theta} E I_{2}=E \int_{Q \times \mathbb{R} \times \mathbb{R}^{d}} \eta^{\prime \prime}\left(u^{\epsilon}-k\right)\left|\nabla G\left(u^{\epsilon}\right)\right|^{2} \varphi \rho_{m}(x-y) \rho_{l}\left[u^{\delta}(t, y)-k\right] d x d t d k d y
$$




$$
+E \int_{Q \times \mathbb{R} \times \mathbb{R}^{d}} \eta^{\prime \prime}\left(u^{\delta}-k\right)\left[\nabla \phi\left(u^{\delta}\right) \nabla u^{\delta}\right] \varphi \rho_{m}(x-y) \rho_{l}\left[u^{\epsilon}(t, x)-k\right] d y d k d x d t
$$

and,

$$
\begin{aligned}
\lim _{n, \theta, l} E I_{2}= & E \int_{Q \times \mathbb{R}^{d}} \eta^{\prime \prime}\left(u^{\epsilon}-u^{\delta}\right)\left|\nabla G\left(u^{\epsilon}\right)\right|^{2} \varphi \rho_{m}(x-y) d x d t d y \\
& +E \int_{Q \times \mathbb{R}^{d}} \eta^{\prime \prime}\left(u^{\delta}-u^{\epsilon}\right)\left[\nabla \phi\left(u^{\delta}\right) \nabla u^{\delta}\right] \varphi \rho_{m}(x-y) d y d x d t \\
= & E \int_{Q \times \mathbb{R}^{d}} \eta^{\prime \prime}\left(u^{\epsilon}-u^{\delta}\right)\left[\left|\nabla G\left(u^{\epsilon}\right)\right|^{2}+\left|\nabla G\left(u^{\delta}\right)\right|^{2}\right] \varphi \rho_{m}(x-y) d x d t d y
\end{aligned}
$$

Now, following the idea of [2], one gets

$$
\begin{aligned}
\tilde{I}_{2} & :=E \int_{Q \times \mathbb{R}^{d}} \eta^{\prime \prime}\left(u^{\epsilon}-u^{\delta}\right)\left[\left|\nabla G\left(u^{\epsilon}\right)\right|^{2}+\left|\nabla G\left(u^{\delta}\right)\right|^{2}\right] \varphi \rho_{m}(x-y) d x d t d y \\
& \geq 2 E \int_{Q \times \mathbb{R}^{d}} \eta^{\prime \prime}\left(u^{\epsilon}(t, x)-u^{\delta}(t, y)\right) \nabla G\left(u^{\epsilon}\right) \cdot \nabla G\left(u^{\delta}\right) \varphi \rho_{m}(x-y) d y d x d t \\
& =2 E \int_{Q \times \mathbb{R}^{d}} \eta^{\prime \prime}\left(u^{\epsilon}(t, x)-u^{\delta}(t, y)\right) \sqrt{\phi^{\prime}\left(u^{\delta}\right)} \nabla_{x} G\left(u^{\epsilon}\right) \cdot \nabla_{y} u^{\delta} \varphi \rho_{m}(x-y) d y d x d t \\
& =2 E \int_{Q \times \mathbb{R}^{d}} \nabla_{x} G\left(u^{\epsilon}\right) \cdot \nabla_{y} \Psi\left(u^{\epsilon}, u^{\delta}\right) \varphi \rho_{m}(x-y) d y d x d t:=\widehat{I}_{2}
\end{aligned}
$$

where $\Psi(a, b)=\int_{a}^{b} \eta^{\prime \prime}(a-\sigma) \sqrt{\phi^{\prime}(\sigma)} d \sigma$. Thus,

$$
\tilde{I}_{2} \geq \widehat{I}_{2}=-2 E \int_{Q \times \mathbb{R}^{d}} \Psi\left(u^{\epsilon}, u^{\delta}\right) \nabla_{x} G\left(u^{\epsilon}\right) \cdot \nabla_{y}\left[\varphi \rho_{m}(x-y)\right] d y d x d t .
$$

Note that, for a fixed $b$, one has that $|\Psi(a, b)| \leq \sqrt{\left\|\phi^{\prime}\right\|_{\infty}} \eta^{\prime}(|a-b|)$ is bounded by assumptions and

$$
\begin{aligned}
\left|\Psi(a, b)-\Psi\left(a_{0}, b\right)\right| \leq & \left|\int_{a}^{b}\left[\eta^{\prime \prime}\left(\sigma-a_{0}\right)-\eta^{\prime \prime}(\sigma-b)\right] \sqrt{\phi^{\prime}(\sigma)} d \sigma\right| \\
& +\left|\int_{a_{0}}^{a} \eta^{\prime \prime}\left(\sigma-a_{0}\right) \sqrt{\phi^{\prime}(\sigma)} d \sigma\right| \\
\leq & C(1+|a-b|)\left|a-a_{0}\right| .
\end{aligned}
$$

Thus, for a fixed $b, a \mapsto \Psi(a, b)$ is a continuous and bounded function, so, Lemma 3 and Green's formula yield:

$$
\begin{aligned}
\widehat{I_{2}} & =-2 E \int_{Q \times \mathbb{R}^{d}} \nabla_{x}\left[\int_{u^{\delta}}^{u^{\epsilon}} \Psi\left(\mu, u^{\delta}\right) \sqrt{\phi^{\prime}(\mu)} d \mu\right] \cdot \nabla_{y}\left[\varphi \rho_{m}(x-y)\right] d y d x d t \\
& =2 E \int_{Q \times \mathbb{R}^{d}}\left[\int_{u^{\delta}}^{u^{\epsilon}} \Psi\left(\mu, u^{\delta}\right) \sqrt{\phi^{\prime}(\mu)} d \mu\right] \operatorname{div}_{x} \nabla_{y}\left[\varphi \rho_{m}(x-y)\right] d y d x d t \\
& =2 E \int_{Q \times \mathbb{R}^{d}}\left[\int_{u^{\delta}}^{u^{\epsilon}} \int_{\mu}^{u^{\delta}} \eta^{\prime \prime}(\mu-\sigma) \sqrt{\phi^{\prime}(\sigma)} d \sigma \sqrt{\phi^{\prime}(\mu)} d \mu\right] \operatorname{div}_{x} \nabla_{y}\left[\varphi \rho_{m}(x-y)\right] d y d x d t .
\end{aligned}
$$


In the sequel, we pass to the limit with $\delta$ and $\epsilon$ to zero in the sense of Young measures as in [1]. This Young measure can be written as a function of the same variables, plus an additional one living in $(0,1)$. To keep in mind the origin of the sequence, we denote by $u_{1}(\cdot, \delta)$ the first limit and by $u_{2}(\cdot, \epsilon)$ the second one.

$$
\begin{aligned}
\lim _{\delta, \epsilon} \widehat{I}_{2}=2 E \int_{Q \times \mathbb{R}^{d} \times(0,1)^{2}}\left[\int_{u_{1}(t, y, \delta)}^{u_{2}(t, x, \epsilon)} \int_{\mu}^{u_{1}(t, y, \delta)}\right. & \left.\eta^{\prime \prime}(\mu-\sigma) \sqrt{\phi^{\prime}(\sigma)} d \sigma \sqrt{\phi^{\prime}(\mu)} d \mu\right] \\
& \times \operatorname{div}_{x} \nabla_{y}\left[\varphi \rho_{m}(x-y)\right] d \epsilon d \delta d y d t d x .
\end{aligned}
$$

3) Next, let us consider

$$
\begin{aligned}
I_{3}:= & -\epsilon \int_{Q \times \mathbb{R} \times Q} \eta^{\prime}\left(u^{\epsilon}-k\right) \nabla u^{\epsilon} \nabla_{x} \psi \rho_{l}\left[u_{\theta}^{\delta}(s, y)-k\right] d x d t d k d y d s \\
& -\delta \int_{Q \times \mathbb{R} \times Q} \eta^{\prime}\left(u_{\theta}^{\delta}-k\right) \nabla u_{\theta}^{\delta} \nabla_{y} \psi \rho_{l}\left[u^{\epsilon}(t, x)-k\right] d y d s d k d x d t . \\
\left|I_{3}\right| \leq & \epsilon\left|\int_{Q \times \mathbb{R} \times Q} \eta^{\prime}\left(u^{\epsilon}-k\right) \nabla u^{\epsilon} \nabla_{x} \psi \rho_{l}\left[u_{\theta}^{\delta}(s, y)-k\right] d x d t d k d y d s\right| \\
& +\delta\left|\int_{Q \times \mathbb{R} \times Q} \eta^{\prime}\left(u_{\theta}^{\delta}-k\right) \nabla u_{\theta}^{\delta} \nabla_{y} \psi \rho_{l}\left[u^{\epsilon}(t, x)-k\right] d y d s d k d x d t\right| \\
\leq & \epsilon \int_{Q \times \mathbb{R} \times Q}\left|\nabla u^{\epsilon} \nabla_{x} \psi\right| \rho_{l}\left[u_{\theta}^{\delta}(s, y)-k\right] d x d t d k d y d s \\
& +\delta \int_{Q \times \mathbb{R} \times Q}\left|\nabla u_{\theta}^{\delta} \nabla_{y} \psi\right| \rho_{l}\left[u^{\epsilon}(t, x)-k\right] d y d s d k d x d t \\
= & \epsilon \int_{Q \times Q}\left|\nabla u^{\epsilon} \nabla_{x} \psi\right| d x d t d y d s+\delta \int_{Q \times Q}\left|\nabla u_{\theta}^{\delta} \nabla_{y} \psi\right| d y d s d x d t \\
\leq & \epsilon \int_{Q}\left|\nabla u^{\epsilon}(t, x)\right| \int_{Q}\left|\nabla_{x} \psi(t, x, s, y)\right| d y d s d x d t \\
& +\delta \int_{Q}\left|\nabla u_{\theta}^{\delta}(s, y)\right| \int_{Q}\left|\nabla_{y} \psi(t, x, s, y)\right| d x d t d y d s
\end{aligned}
$$

Thus, replacing $\psi(t, x, s, y)$ by $\varphi(t, x) \rho_{n}(t-s) \rho_{m}(x-y)$,

$$
\begin{aligned}
\left|E I_{3}\right| \leq & E\left|I_{3}\right| \\
\leq & \epsilon E \int_{K}\left|\nabla u^{\epsilon}(t, x)\right| \int_{Q} \rho_{n}(t-s)\left|\varphi \nabla_{x} \rho_{m}(x-y)+\rho_{m}(x-y) \nabla \varphi\right| d y d s d x d t \\
& \quad+\delta E \int_{K}\left|\nabla u_{\theta}^{\delta}(s, y)\right| \int_{Q} \rho_{n}(t-s)\left|\varphi \nabla_{y} \rho_{m}(x-y)\right| d x d t d y d s \\
& \leq C(m, K)\left[\epsilon\left\|\nabla u^{\epsilon}\right\|_{L^{2}(\Omega \times Q)}+\delta\left\|\nabla u^{\delta}\right\|_{L^{2}(\Omega \times Q)}\right] \leq C(m, K)[\sqrt{\epsilon}+\sqrt{\delta}]
\end{aligned}
$$

thanks to the a priori estimates (see Lemma 4).

Therefore, $\lim _{n, \theta, l, \delta, \epsilon} E I_{3}=0$. 
4) Now let us consider the integrals coming from the initial conditions, i.e.

$$
\begin{aligned}
I_{4}:= & \int_{Q \times \mathbb{R} \times \mathbb{R}^{d}} H\left(u_{0}^{\epsilon}, k\right) \psi(t=0) \rho_{l}\left[u_{\theta}^{\delta}(s, y)-k\right] d x d k d y d s \\
& +\int_{Q \times \mathbb{R} \times \mathbb{R}^{d}} H\left(u_{0, \theta}^{\delta}, k\right) \psi(s=0) \rho_{l}\left[u^{\epsilon}(t, x)-k\right] d y d k d x d t .
\end{aligned}
$$

If $\psi(t, x, s, y)=\varphi(t, x) \rho_{n}(t-s) \rho_{m}(x-y)$, then

$$
\begin{aligned}
I_{4}= & \int_{Q \times \mathbb{R} \times \mathbb{R}^{d}} H\left(u_{0}^{\epsilon}, k\right) \varphi(0, x) \rho_{n}(-s) \rho_{m}(x-y) \rho_{l}\left[u_{\theta}^{\delta}(s, y)-k\right] d x d k d y d s \\
& +\int_{Q \times \mathbb{R} \times \mathbb{R}^{d}} H\left(u_{0, \theta}^{\delta}, k\right) \varphi \rho_{n}(t) \rho_{m}(x-y) \rho_{l}\left[u^{\epsilon}(t, x)-k\right] d y d k d x d t \\
= & \int_{Q \times \mathbb{R} \times \mathbb{R}^{d}} H\left(u_{0}^{\epsilon}, k\right) \varphi(0, x) \rho_{n}(-s) \rho_{m}(x-y) \rho_{l}\left[u_{\theta}^{\delta}(s, y)-k\right] d x d k d y d s
\end{aligned}
$$

as supp $\rho_{n} \subset[-2 / n, 0]$, and then a slight modification of similar arguments in [1] yields

$$
\lim _{n, \theta, l, \delta, \epsilon} E I_{4}=\int_{\mathbb{R}^{2 d}} \varphi(0, x) \eta\left(u_{0,1}-u_{0,2}\right) \rho_{m}(x-y) d x d y .
$$

5) Consider now

$$
\begin{aligned}
I_{5}:= & \int_{Q \times \mathbb{R} \times Q}\left(H\left(u^{\epsilon}, k\right) \partial_{t} \psi+\phi^{\eta}\left(u^{\epsilon}, k\right) \Delta_{x} \psi-F^{\eta}\left(u^{\epsilon}, k\right) \nabla_{x} \psi\right) \\
& \times \rho_{l}\left[u_{\theta}^{\delta}(s, y)-k\right] d x d t d k d y d s \\
& +\int_{Q \times \mathbb{R} \times Q}\left(H\left(u_{\theta}^{\delta}, k\right) \partial_{s} \psi-\eta^{\prime}\left(u_{\theta}^{\delta}-k\right) \nabla \phi\left(u^{\delta}\right)_{\theta} \nabla_{y} \psi-\eta^{\prime}\left(u_{\theta}^{\delta}-k\right)\left[\vec{f}\left(u^{\delta}\right)_{\theta} \nabla_{y} \psi\right]\right) \\
& \times \rho_{l}\left[u^{\epsilon}(t, x)-k\right] d y d s d k d x d t \\
& -\int_{Q \times \mathbb{R} \times Q} \eta^{\prime \prime}\left(u_{\theta}^{\delta}-k\right)\left[\vec{f}\left(u^{\delta}\right)_{\theta} \nabla u_{\theta}^{\delta}\right] \psi \rho_{l}\left[u^{\epsilon}(t, x)-k\right] d y d s d k d x d t
\end{aligned}
$$

Since $H(x, k)=\eta(x-k)$ with an even function $\eta$,

$$
\begin{aligned}
& I_{5}= \int_{Q \times \mathbb{R} \times Q}\left(H\left(u^{\epsilon}, u_{\theta}^{\delta}(s, y)-\zeta\right) \partial_{t} \psi+\phi^{\eta}\left(u^{\epsilon}, u_{\theta}^{\delta}(s, y)-\zeta\right) \Delta_{x} \psi\right. \\
&\left.-F^{\eta}\left(u^{\epsilon}, u_{\theta}^{\delta}(s, y)-\zeta\right) \nabla_{x} \psi\right) \rho_{l}[\zeta] d x d t d \zeta d y d s \\
& \quad+\int_{Q \times \mathbb{R} \times Q}\left(H\left(u_{\theta}^{\delta}, u^{\epsilon}(t, x)+\zeta\right) \partial_{s} \psi-\eta^{\prime}\left(u_{\theta}^{\delta}-u^{\epsilon}(t, x)-\zeta\right) \nabla \phi\left(u^{\delta}\right)_{\theta} \nabla_{y} \psi\right. \\
&\left.-\eta^{\prime}\left(u_{\theta}^{\delta}-u^{\epsilon}(t, x)-\zeta\right)\left[\vec{f}\left(u^{\delta}\right)_{\theta} \nabla_{y} \psi\right]\right) \rho_{l}\left[u^{\epsilon}(t, x)-u^{\epsilon}(t, x)-\zeta\right] d y d s d \zeta d x d t \\
& \quad-\int_{Q^{2} \times \mathbb{R}} \eta^{\prime \prime}\left(u_{\theta}^{\delta}-u^{\epsilon}(t, x)-\zeta\right)\left[\vec{f}\left(u^{\delta}\right)_{\theta} \nabla u_{\theta}^{\delta}\right] \psi \rho_{l}\left[u^{\epsilon}(t, x)-u^{\epsilon}(t, x)-\zeta\right] d y d s d \zeta d x d t
\end{aligned}
$$


Replacing $\psi(t, s, x, y)$ by $\varphi(t, x) \rho_{n}(t-s) \rho_{m}(x-y)$, one gets

$$
\begin{gathered}
I_{5}=\int_{Q \times \mathbb{R} \times Q} \begin{array}{r}
H\left(u^{\epsilon}, u_{\theta}^{\delta}(s, y)-\zeta\right)\left[\partial_{t} \varphi\right] \rho_{n}(t-s) \rho_{m}(x-y) \\
+\phi^{\eta}\left(u^{\epsilon}, u_{\theta}^{\delta}(s, y)-\zeta\right) \Delta_{x}\left[\varphi \rho_{n}(t-s) \rho_{m}(x-y)\right] \\
\left.-F^{\eta}\left(u^{\epsilon}, u_{\theta}^{\delta}(s, y)-\zeta\right) \nabla_{x}\left[\varphi \rho_{n}(t-s) \rho_{m}(x-y)\right]\right) \rho_{l}[\zeta] d x d t d \zeta d y d s \\
-\int_{Q \times \mathbb{R} \times Q}\left(\eta^{\prime}\left(u_{\theta}^{\delta}-u^{\epsilon}(t, x)-\zeta\right) \nabla \phi\left(u^{\delta}\right)_{\theta} \nabla_{y}\left[\varphi \rho_{n}(t-s) \rho_{m}(x-y)\right]\right. \\
\left.+\eta^{\prime}\left(u_{\theta}^{\delta}-u^{\epsilon}(t, x)-\zeta\right)\left[\vec{f}\left(u^{\delta}\right)_{\theta} \nabla_{y}\left[\varphi \rho_{n}(t-s) \rho_{m}(x-y)\right]\right]\right) \rho_{l}[\zeta] d y d s d \zeta d x d t \\
-\int_{Q^{2} \times \mathbb{R}} \eta^{\prime \prime}\left(u_{\theta}^{\delta}-u^{\epsilon}(t, x)-\zeta\right)\left[\vec{f}\left(u^{\delta}\right)_{\theta} \nabla u_{\theta}^{\delta}\right]\left[\varphi \rho_{n}(t-s) \rho_{m}(x-y)\right] \rho_{l}[\zeta] d y d s d \zeta d x d t
\end{array}
\end{gathered}
$$

Thus, passing to the limit with respect to $n$,

$$
\begin{aligned}
\lim _{n} E I_{5}=E \int_{Q \times \mathbb{R} \times \mathbb{R}^{d}}\left(\begin{array}{r}
H\left(u^{\epsilon}, u_{\theta}^{\delta}(t, y)-\zeta\right)\left[\partial_{t} \varphi\right] \rho_{m}(x-y) \\
+\phi^{\eta}\left(u^{\epsilon}, u_{\theta}^{\delta}(t, y)-\zeta\right) \Delta_{x}\left[\varphi \rho_{m}(x-y)\right] \\
\left.-F^{\eta}\left(u^{\epsilon}, u_{\theta}^{\delta}(t, y)-\zeta\right) \nabla_{x}\left[\varphi \rho_{m}(x-y)\right]\right) \rho_{l}[\zeta] d x d t d \zeta d y
\end{array}\right. \\
-E \int_{Q \times \mathbb{R} \times \mathbb{R}^{d}}\left(\eta^{\prime}\left(u_{\theta}^{\delta}-u^{\epsilon}(t, x)-\zeta\right) \nabla \phi\left(u^{\delta}\right)_{\theta} \nabla_{y}\left[\varphi \rho_{m}(x-y)\right]\right. \\
\left.+\eta^{\prime}\left(u_{\theta}^{\delta}-u^{\epsilon}(t, x)-\zeta\right)\left[\vec{f}\left(u^{\delta}\right)_{\theta} \nabla_{y}\left[\varphi \rho_{m}(x-y)\right]\right]\right) \rho_{l}[\zeta] d y d \zeta d x d t \\
-E \int_{Q \times \mathbb{R} \times \mathbb{R}^{d}} \eta^{\prime \prime}\left(u_{\theta}^{\delta}-u^{\epsilon}(t, x)-\zeta\right)\left[\vec{f}\left(u^{\delta}\right)_{\theta} \nabla u_{\theta}^{\delta}\right]\left[\varphi \rho_{m}(x-y)\right] \rho_{l}[\zeta] d y d \zeta d x d t
\end{aligned}
$$

and, passing to the limit with respect to $\theta$,

$$
\begin{aligned}
& \lim _{n, \theta} E I_{5}=E \int_{Q \times \mathbb{R} \times \mathbb{R}^{d}}\left(H\left(u^{\epsilon}, u^{\delta}(t, y)-\zeta\right)\left[\partial_{t} \varphi\right] \rho_{m}(x-y)\right. \\
& +\phi^{\eta}\left(u^{\epsilon}, u^{\delta}(t, y)-\zeta\right) \Delta_{x}\left[\varphi \rho_{m}(x-y)\right] \\
& \left.-F^{\eta}\left(u^{\epsilon}, u^{\delta}(t, y)-\zeta\right) \nabla_{x}\left[\varphi \rho_{m}(x-y)\right]\right) \rho_{l}[\zeta] d x d t d \zeta d y \\
& -E \int_{Q \times \mathbb{R} \times \mathbb{R}^{d}}\left(\eta^{\prime}\left(u^{\delta}-u^{\epsilon}(t, x)-\zeta\right) \nabla \phi\left(u^{\delta}\right) \nabla_{y}\left[\varphi \rho_{m}(x-y)\right]\right. \\
& \left.+\eta^{\prime}\left(u^{\delta}-u^{\epsilon}(t, x)-\zeta\right)\left[\vec{f}\left(u^{\delta}\right) \nabla_{y}\left[\varphi \rho_{m}(x-y)\right]\right]\right) \rho_{l}[\zeta] d y d \zeta d x d t \\
& -E \int_{Q \times \mathbb{R} \times \mathbb{R}^{d}} \eta^{\prime \prime}\left(u^{\delta}-u^{\epsilon}(t, x)-\zeta\right)\left[\vec{f}\left(u^{\delta}\right) \nabla u^{\delta}\right]\left[\varphi \rho_{m}(x-y)\right] \rho_{l}[\zeta] d y d \zeta d x d t
\end{aligned}
$$

Then, formulas of Green's type give

$$
\lim _{n, \theta, l} E I_{5}=
$$




$$
\begin{gathered}
E \int_{Q \times \mathbb{R}^{d}}\left(\eta\left(u^{\epsilon}-u^{\delta}\right)\left[\partial_{t} \varphi\right] \rho_{m}(x-y)\right) d x d t d y \\
+\quad E \int_{Q \times \mathbb{R}^{d}}\left(\phi^{\eta}\left(u^{\epsilon}, u^{\delta}(t, y)\right) \Delta_{x}\left[\varphi \rho_{m}(x-y)\right]+\phi^{\eta}\left(u^{\delta}, u^{\epsilon}(t, x)\right) \Delta_{y}\left[\varphi \rho_{m}(x-y)\right]\right) d y d x d t \\
-\quad E \int_{Q \times \mathbb{R}^{d}}\left(F^{\eta}\left(u^{\epsilon}, u^{\delta}(t, y)\right) \nabla_{x}\left[\varphi \rho_{m}(x-y)\right]+F^{\eta}\left(u^{\delta}, u^{\epsilon}(t, x)\right) \nabla_{y}\left[\varphi \rho_{m}(x-y)\right]\right) d y d x d t .
\end{gathered}
$$

Passing to the limits with respect to $\delta$ and $\epsilon$ gives

$$
\begin{aligned}
\lim _{n, \theta, l, \delta, \epsilon} E I_{5}= & E \int_{Q \times \mathbb{R}^{d} \times(0,1)^{2}} \eta\left[u_{2}(t, x, \epsilon)-u_{1}(t, y, \delta)\right] \partial_{t} \varphi \rho_{m}(x-y) d \epsilon d \delta d x d t d y \\
& +E \int_{Q \times \mathbb{R}^{d} \times(0,1)^{2}}\left(\phi^{\eta}\left(u_{2}(t, x, \epsilon), u_{1}(t, y, \delta)\right) \Delta_{x}\left[\varphi \rho_{m}(x-y)\right]\right. \\
& \left.+\phi^{\eta}\left(u_{1}(t, y, \delta), u_{2}(t, x, \epsilon)\right) \Delta_{y}\left[\varphi \rho_{m}(x-y)\right]\right) d \epsilon d \delta d y d x d t \\
& -E \int_{Q \times \mathbb{R}^{d} \times(0,1)^{2}}\left(F^{\eta}\left(u_{2}(t, x, \epsilon), u_{1}(t, y, \delta)\right) \nabla_{x}\left[\varphi \rho_{m}(x-y)\right]\right. \\
& \left.+F^{\eta}\left(u_{1}(t, y, \delta), u_{2}(t, x, \epsilon)\right) \nabla_{y}\left[\varphi \rho_{m}(x-y)\right]\right) d \epsilon d \delta d y d x d t .
\end{aligned}
$$

6) Let us now consider the additional deterministic integrals coming from the Itô integral formula:

$$
\begin{aligned}
I_{6}:= & \frac{1}{2} \int_{Q \times \mathbb{R} \times Q} \eta^{\prime \prime}\left(u^{\epsilon}-k\right)\left[h\left(x, u^{\epsilon}\right)\right]^{2} \psi \rho_{l}\left[u_{\theta}^{\delta}(s, y)-k\right] d t d x d k d y d s \\
& +\frac{1}{2} \int_{Q \times \mathbb{R} \times Q} \eta^{\prime \prime}\left(u_{\theta}^{\delta}-k\right)\left[h\left(y, u^{\delta}\right)_{\theta}\right]^{2} \psi \rho_{l}\left[u^{\epsilon}(t, x)-k\right] d y d s d k d x d t .
\end{aligned}
$$

Passing to the limit with respect to $n, \theta$, then $l$, one obtains

$\lim _{n, \theta, l} E I_{6}=\frac{1}{2} E \int_{Q \times \mathbb{R}^{d}}\left(\left|h\left(x, u^{\epsilon}\right)\right|^{2}+\left|h\left(y, u^{\delta}\right)\right|^{2}\right) \varphi \rho_{m}(x-y) \eta^{\prime \prime}\left(u^{\epsilon}-u^{\delta}\right) d x d t d y$

Then, like in [1], we need to add this term to the one in item 7).

7) Now let us consider the stochastic Itô integral terms:

$$
\begin{aligned}
I_{7}:= & \int_{Q \times \mathbb{R} \times Q} \eta^{\prime}\left(u^{\epsilon}-k\right) \psi h\left(x, u^{\epsilon}\right) d w(t) \rho_{l}\left[u_{\theta}^{\delta}(s, y)-k\right] d x d k d y d s \\
& +\int_{Q \times \mathbb{R} \times Q} \eta^{\prime}\left(u_{\theta}^{\delta}-k\right) \psi h\left(y, u^{\delta}\right)_{\theta} d w(s) \rho_{l}\left[u^{\epsilon}(t, x)-k\right] d y d k d x d t
\end{aligned}
$$

Taking the expectation, replacing $\psi(t, s, x, y)$ by $\varphi(t, x) \rho_{n}(t-s) \rho_{m}(x-y)$ and since the support of $\rho_{n}$ is negative, as already remarked in [1], the second integral vanishes and one gets that

$$
E I_{7}=E \int_{Q \times \mathbb{R} \times Q} \eta^{\prime}\left(u^{\epsilon}-k\right) \psi h\left(x, u^{\epsilon}\right) d w(t) \rho_{l}\left[u_{\theta}^{\delta}(s, y)-k\right] d x d k d y d s
$$




$$
\begin{gathered}
=E \int_{Q} \int_{\mathbb{R}} \int_{\mathbb{R}^{d}} \int_{s-2 / n}^{s} \eta^{\prime}\left(u^{\epsilon}(t, x)-k\right) \varphi(t, x) h\left(x, u^{\epsilon}(t, x)\right) \rho_{n}(t-s) d w(t) \\
\times \rho_{m}(x-y) \rho_{l}\left[u_{\theta}^{\delta}(s, y)-k\right] d x d k d y d s \\
=E \int_{Q} \int_{\mathbb{R}} \int_{\mathbb{R}^{d}} \mathcal{F}(x, s, k) \rho_{m}(x-y) \rho_{l}\left[u_{\theta}^{\delta}(s, y)-k\right] d x d k d y d s \\
=-E \int_{Q} \int_{\mathbb{R}} \int_{\mathbb{R}^{d}} \mathcal{F}(x, s, k) \rho_{m}(x-y) \frac{\partial}{\partial k} \operatorname{Sgn}_{l}\left[u_{\theta}^{\delta}(s, y)-k\right] d x d k d y d s \\
=E \int_{Q} \int_{\mathbb{R}} \int_{\mathbb{R}^{d}} \frac{\partial}{\partial k} \mathcal{F}(x, s, k) \rho_{m}(x-y) \operatorname{Sgn}_{l}\left[u_{\theta}^{\delta}(s, y)-k\right] d x d k d y d s \\
=E \int_{0}^{T} \int_{\mathbb{R}} \int_{\mathbb{R}^{d}} \frac{\partial}{\partial k} \mathcal{F}(x, s, k) \int_{\mathbb{R}^{d}}\left(\operatorname{Sgn}_{l}\left[u_{\theta}^{\delta}(s, y)-k\right]-\operatorname{Sgn}_{l}\left[u_{\theta}^{\delta}(s-2 / n, y)-k\right]\right) \\
\times \rho_{m}(x-y) d y d x d k d s,
\end{gathered}
$$

where, for convenience, one denotes by $\operatorname{Sgn}_{l}$ an antiderivative of $\rho_{l}$ and

$$
\mathcal{F}(x, s, k)=\int_{s-2 / n}^{s} \eta^{\prime}\left(u^{\epsilon}(t, x)-k\right) \varphi(t, x) h\left(x, u^{\epsilon}(t, x)\right) \rho_{n}(t-s) d w(t) .
$$

Thanks to Itô's formula, if one denotes by $\vec{A}_{\delta}(s, y)=\delta \nabla u_{\theta}^{\delta}+\nabla \phi\left(u^{\delta}\right)_{\theta}+$ $\vec{f}\left(u^{\delta}\right)_{\theta}$ (remind : $\left.d u_{\theta}^{\delta}-\operatorname{div} \vec{A}_{\delta} d t=g\left(y, u^{\delta}\right)_{\theta} d t+h\left(y, u^{\delta}\right)_{\theta} d w\right)$, we find

$$
\begin{aligned}
& \left(\operatorname{Sgn}_{l}\left[u_{\theta}^{\delta}(s, y)-k\right]-\operatorname{Sgn}_{l}\left[u_{\theta}^{\delta}(s-2 / n, y)-k\right]\right) \rho_{m}(x-y) \\
& \left.=\int_{s-2 / n}^{s} \operatorname{div} \vec{A}_{\delta}\left[\operatorname{Sgn}_{l}^{\prime}\left[u_{\theta}^{\delta}(\sigma, y)-k\right] \rho_{m}(x-y)\right]\right] d \sigma \\
& +\frac{1}{2} \int_{s-2 / n}^{s} \operatorname{Sgn}_{l}^{\prime \prime}\left[u_{\theta}^{\delta}(\sigma, y)-k\right] \rho_{m}(x-y)\left(h\left(y, u^{\delta}\right)_{\theta}\right)^{2} d \sigma \\
& \quad+\int_{s-2 / n}^{s} \operatorname{Sgn}_{l}^{\prime}\left[u_{\theta}^{\delta}(\sigma, y)-k\right] \rho_{m}(x-y) h\left(y, u^{\delta}\right)_{\theta} d w(\sigma)
\end{aligned}
$$

Since $\frac{\partial}{\partial k} \mathcal{F}(x, s, k)=-\int_{s-2 / n}^{s} \eta^{\prime \prime}\left(u^{\epsilon}(t, x)-k\right) \varphi(t, x) h\left(x, u^{\epsilon}(t, x)\right) \rho_{n}(t-s) d w(t)$

(thanks to [17](Theorem 7.6, p. 180)), following [1], one gets that

$$
\lim _{n, \theta, l} E I_{7}=-E \int_{Q \times \mathbb{R}^{d}} \eta^{\prime \prime}\left(u^{\epsilon}-u^{\delta}\right) h\left(x, u^{\epsilon}\right) h\left(y, u^{\delta}\right) \rho_{m}(x-y) d x d t d y .
$$

Therefore,

$$
\begin{aligned}
& \lim _{n, \theta, l} E I_{6}+\lim _{n, \theta, l} E I_{7} \\
= & \frac{1}{2} E \int_{Q \times \mathbb{R}^{d}}\left(\left|h\left(x, u^{\epsilon}\right)\right|^{2}+\left|h\left(y, u^{\delta}\right)\right|^{2}\right) \varphi \rho_{m}(x-y) \eta^{\prime \prime}\left(u^{\epsilon}-u^{\delta}\right) d x d t d y \\
& -E \int_{Q \times \mathbb{R}^{d}} \eta^{\prime \prime}\left(u^{\epsilon}-u^{\delta}\right) h\left(x, u^{\epsilon}\right) h\left(y, u^{\delta}\right) \rho_{m}(x-y) d x d t d y \\
= & \frac{1}{2} E \int_{Q \times \mathbb{R}^{d}}\left|h\left(x, u^{\epsilon}\right)-h\left(y, u^{\delta}\right)\right|^{2} \varphi \rho_{m}(x-y) \eta^{\prime \prime}\left(u^{\epsilon}-u^{\delta}\right) d x d t d y,
\end{aligned}
$$


and

$$
\begin{aligned}
& \lim _{\delta, \epsilon}\left[\lim _{n, \theta, l} E I_{6}+\lim _{n, \theta, l} E I_{7}\right] \\
& =\frac{1}{2} E \int_{Q \times \mathbb{R}^{d} \times(0,1)^{2}}\left|h\left(x, u_{2}(t, x, \epsilon)\right)-h\left(y, u_{1}(t, y, \delta)\right)\right|^{2} \varphi \rho_{m}(x-y) \\
& \times \eta^{\prime \prime}\left(u_{2}(t, x, \epsilon)-u_{1}(t, y, \delta)\right) d \epsilon d \delta d x d t d y .
\end{aligned}
$$

8) Finally, let us consider the reaction terms:

$$
\begin{aligned}
I_{8}:=\int_{\mathbb{R} \times Q^{2}}\left(\eta^{\prime}\left(u^{\epsilon}-k\right) \psi g\left(x, u^{\epsilon}\right) \rho_{l}\left[u_{\theta}^{\delta}(s, y)-k\right]\right. \\
\left.\quad+\eta^{\prime}\left(u_{\theta}^{\delta}-k\right) \psi g\left(y, u^{\delta}\right)_{\theta} \rho_{l}\left[u^{\epsilon}(t, x)-k\right]\right) d y d s d k d x d t .
\end{aligned}
$$

Classical convergence arguments for integrals yield

$$
\begin{aligned}
\lim _{n, \theta, l} E I_{8} & =E \int_{\mathbb{R}^{d} \times Q} \eta^{\prime}\left(u^{\epsilon}-u^{\delta}\right) \varphi\left[g\left(x, u^{\epsilon}\right)-g\left(y, u^{\delta}\right)\right] \rho_{m}(x-y) d y d x d t \\
& \leq E \int_{\mathbb{R}^{d} \times Q} \varphi\left|g\left(x, u^{\epsilon}\right)-g\left(y, u^{\delta}\right)\right| \rho_{m}(x-y) d y d x d t,
\end{aligned}
$$

and

$$
\begin{aligned}
& \lim \sup _{\delta, \epsilon} \lim _{n, \theta, l} E I_{8} \\
\leq & E \int_{\mathbb{R}^{d} \times Q \times(0,1)^{2}} \varphi\left|g\left(x, u_{2}(t, x, \epsilon)\right)-g\left(y, u_{1}(t, y, \delta)\right)\right| \rho_{m}(x-y) d \delta d \epsilon d y d x d t .
\end{aligned}
$$

Coming back to the contributions, we started with

$$
I_{1}+I_{2}=I_{3}+I_{4}+I_{5}+I_{6}+I_{7}+I_{8}
$$

to get, in a first step

$$
E I_{2} \leq C(m, K)[\sqrt{\epsilon}+\sqrt{\delta}]+E I_{4}+E I_{5}+E I_{6}+E I_{7}+E I_{8} .
$$

Then we can estimate

$$
\begin{aligned}
\widehat{I}_{2} & \leq \tilde{I}_{2}=\lim _{n, \theta, l} E I_{2} \\
& \leq C(m, K)[\sqrt{\epsilon}+\sqrt{\delta}]+\lim _{n, \theta, l} E I_{4}+\lim _{n, \theta, l} E I_{5}+\lim _{n, \theta, l} E I_{6}+\lim _{n, \theta, l} E I_{7}+\lim _{n, \theta, l} E I_{8},
\end{aligned}
$$

which gives, as $\epsilon$ and $\delta$ tend to zero,

$$
2 E \int_{Q \times \mathbb{R}^{d} \times(0,1)^{2}}\left[\int_{u_{1}(t, y, \delta)}^{u_{2}(t, x, \epsilon)} \int_{\mu}^{u_{1}(t, y, \delta)} \eta^{\prime \prime}(\mu-\sigma) \sqrt{\phi^{\prime}(\sigma)} d \sigma \sqrt{\phi^{\prime}(\mu)} d \mu\right]
$$


$\times \operatorname{div}_{x} \nabla_{y}\left[\varphi \rho_{m}(x-y)\right] d \epsilon d \delta d y d t d x$

$$
\begin{aligned}
= & \lim _{\delta, \epsilon} \widehat{I}_{2} \leq \lim _{n, \theta, l, \delta, \epsilon} E I_{4}+\lim _{n, \theta, l, \delta, \epsilon} E I_{5}+\lim _{\delta, \epsilon}\left[\lim _{n, \theta, l} E I_{6}+\lim _{n, \theta, l} E I_{7}\right]+\lim _{\delta, \epsilon} \sup _{n, \theta, l} \lim _{\leq} E I_{8} \\
\leq & \int_{\mathbb{R}^{2 d}} \varphi(0, x) \eta\left(u_{0,1}-u_{0,2}\right) \rho_{m}(x-y) d x d y \\
& +E \int_{Q \times \mathbb{R}^{d} \times(0,1)^{2}} \eta\left[u_{2}(t, x, \epsilon)-u_{1}(t, y, \delta)\right] \partial_{t} \varphi \rho_{m}(x-y) d \epsilon d \delta d x d t d y \\
& +E \int_{Q \times \mathbb{R}^{d} \times(0,1)^{2}}\left(\phi^{\eta}\left(u_{2}(t, x, \epsilon), u_{1}(t, y, \delta)\right) \Delta_{x}\left[\varphi \rho_{m}(x-y)\right]\right. \\
& +E \int_{Q \times \mathbb{R}^{d} \times(0,1)^{2}}\left(F^{\eta}\left(u_{2}(t, x, \epsilon), u_{1}(t, y, \delta)\right) \nabla_{x}\left[\varphi \rho_{m}(x-y)\right]\right. \\
& +\frac{1}{2} E \int_{Q \times \mathbb{R}^{d} \times(0,1)^{2}}\left|h\left(x, u_{2}(t, x, \epsilon)\right)-h\left(y, u_{1}(t, y, \delta)\right)\right|^{2} \varphi \rho_{m}(x-y) \\
& +E \int_{\mathbb{R}^{d} \times Q \times(0,1)^{2}} \varphi\left|g\left(x, u_{2}(t, x, \epsilon)\right)-g\left(y, u_{1}(t, y, \delta)\right)\right| \rho_{m}(x-y) d \delta d \epsilon d y d x d y d x d t .
\end{aligned}
$$

Developing terms we find

$$
\begin{aligned}
0 \leq & \int_{\mathbb{R}^{2 d}} \varphi(0, x) \eta\left(u_{0,1}-u_{0,2}\right) \rho_{m}(x-y) d x d y \\
& +E \int_{Q \times \mathbb{R}^{d} \times(0,1)^{2}} \eta\left(u_{2}(t, x, \epsilon)-u_{1}(t, y, \delta)\right) \partial_{t} \varphi \rho_{m}(x-y) d \epsilon d \delta d x d t d y \\
& +E \int_{Q \times \mathbb{R}^{d} \times(0,1)^{2}}\left(\phi^{\eta}\left(u_{2}(t, x, \epsilon), u_{1}(t, y, \delta)\right) \Delta \varphi-F^{\eta}\left(u_{2}(t, x, \epsilon), u_{1}(t, y, \delta)\right) \nabla \varphi\right) \\
& +2 E \int_{Q \times \mathbb{R}^{d} \times(0,1)^{2}} \phi^{\eta}\left(u_{2}(t, x, \epsilon), u_{1}(t, y, \delta)\right) \nabla \varphi \nabla \rho_{m}(x-y) d \epsilon d \delta d y d x d t \\
& +E \int_{Q \times \mathbb{R}^{d} \times(0,1)^{2}} \varphi \Delta \rho_{m}(x-y)\left(\phi^{\eta}\left(u_{2}(t, x, \epsilon), u_{1}(t, y, \delta)\right)\right. \\
& \left.+\phi^{\eta}\left(u_{1}(t, y, \delta), u_{2}(t, x, \epsilon)\right)\right) d \epsilon d \delta d y d x d t \\
& -E \int_{Q \times \mathbb{R}^{d} \times(0,1)^{2}} \varphi\left(F^{\eta}\left(u_{2}(t, x, \epsilon), u_{1}(t, y, \delta)\right)-F^{\eta}\left(u_{1}(t, y, \delta), u_{2}(t, x, \epsilon)\right)\right) \\
+ & \times \rho_{Q}(x-y) d \epsilon d \delta d y d x d t \\
& \times \int_{Q \times \mathbb{R}^{d} \times(0,1)^{2}}\left|h\left(x, u_{2}(t, x, \epsilon)\right)-h\left(y, u_{1}(t, y, \delta)\right)\right|^{2} \varphi \rho_{m}(x-y)
\end{aligned}
$$




$$
\begin{array}{r}
\times \eta^{\prime \prime}\left(u_{2}(t, x, \epsilon)-u_{1}(t, y, \delta)\right) d x d t d y \\
+E \int_{\mathbb{R}^{d} \times Q \times(0,1)^{2}} \varphi\left|g\left(x, u_{2}(t, x, \epsilon)\right)-g\left(y, u_{1}(t, y, \delta)\right)\right| \rho_{m}(x-y) d \delta d \epsilon d y d x d t \\
+2 E \int_{Q \times \mathbb{R}^{d} \times(0,1)^{2}}\left[\int_{u_{1}(t, y, \delta)}^{u_{2}(t, x, \epsilon)} \int_{\mu}^{u_{1}(t, y, \delta)} \eta^{\prime \prime}(\mu-\sigma) \sqrt{\phi^{\prime}(\sigma)} d \sigma \sqrt{\phi^{\prime}(\mu)} d \mu\right] \\
\times\left[\nabla \varphi \nabla \rho_{m}(x-y)+\varphi \Delta \rho_{m}(x-y)\right] d \epsilon d \delta d y d t d x .
\end{array}
$$

Then, thanks to Lemma 1 and assumptions on $h$,

$$
\begin{aligned}
& 0 \leq \int_{\mathbb{R}^{2 d}} \varphi(0, x)\left|u_{0,1}-u_{0,2}\right| \rho_{m}(x-y) d x d y \\
& +E \int_{Q \times \mathbb{R}^{d} \times(0,1)^{2}}\left|u_{2}(t, x, \epsilon)-u_{1}(t, y, \delta)\right| \partial_{t} \varphi \rho_{m}(x-y) d \epsilon d \delta d x d t d y \\
& +E \int_{Q \times \mathbb{R}^{d} \times(0,1)^{2}}\left(\left|\phi\left(u_{2}(t, x, \epsilon)\right)-\phi\left(u_{1}(t, y, \delta)\right)\right| \Delta \varphi\right. \\
& \left.-F\left(u_{2}(t, x, \epsilon), u_{1}(t, y, \delta)\right) \nabla \varphi\right) \rho_{m}(x-y) d \epsilon d \delta d y d x d t \\
& +E \int_{\mathbb{R}^{d} \times Q \times(0,1)^{2}} \varphi\left|g\left(x, u_{2}(t, x, \epsilon)\right)-g\left(y, u_{1}(t, y, \delta)\right)\right| \rho_{m}(x-y) d \delta d \epsilon d y d x d t \\
& +\tau \int_{\mathbb{R}^{d}} \varphi(0, x) d x+\tau \int_{Q}\left|\partial_{t} \varphi\right|+c(\phi)|\Delta \varphi|+c(\vec{f})|\nabla \varphi| d x d t \\
& +c(h) E \int_{Q \times \mathbb{R}^{d} \times(0,1)^{2}}\left|u_{2}(t, x)-u_{1}(t, y, \delta)\right|^{2} \varphi \rho_{m}(x-y) \eta^{\prime \prime}\left(u_{2}(t, x, \epsilon)-u_{1}(t, y, \delta)\right) d x d t d y \\
& +c(h) E \int_{Q \times \mathbb{R}^{d} \times(0,1)^{2}}\left|\omega_{h}(\|x-y\|)\right|^{2} \varphi \rho_{m}(x-y) \eta^{\prime \prime}\left(u_{2}(t, x, \epsilon)-u_{1}(t, y, \delta)\right) d x d t d y \\
& -E \int_{Q \times \mathbb{R}^{d} \times(0,1)^{2}} \varphi\left(F^{\eta}\left(u_{2}(t, x, \epsilon), u_{1}(t, y, \delta)\right)-F^{\eta}\left(u_{1}(t, y, \delta), u_{2}(t, x, \epsilon)\right)\right) \\
& \times \nabla \rho_{m}(x-y) d \epsilon d \delta d y d x d t \\
& +2 E \int_{Q \times \mathbb{R}^{d} \times(0,1)^{2}} \phi^{\eta}\left(u_{2}(t, x, \epsilon), u_{1}(t, y, \delta)\right) \nabla \varphi \nabla \rho_{m}(x-y) d \epsilon d \delta d y d x d t \\
& +E \int_{Q \times \mathbb{R}^{d} \times(0,1)^{2}}^{\varphi \Delta \rho_{m}}(x-y)\left(\phi^{\eta}\left(u_{2}(t, x, \epsilon), u_{1}(t, y, \delta)\right)+\phi^{\eta}\left(u_{1}(t, y, \delta), u_{2}(t, x, \epsilon)\right)\right) \\
& \times d \epsilon d \delta d y d x d t \\
& +2 E \int_{Q \times \mathbb{R}^{d} \times(0,1)^{2}} I_{\tau^{2}}\left(u_{1}(t, y), u_{2}(t, x, \epsilon)\right)\left[\nabla \varphi \nabla \rho_{m}(x-y)+\varphi \Delta \rho_{m}(x-y)\right] d \epsilon d \delta d y d t d x \\
& =A_{1}+A_{2}+A_{3}+A_{4}+A_{5}+A_{6}+A_{7}+A_{8}+A_{9}+A_{10}
\end{aligned}
$$

where one sets, for any $a, b$,

$$
I_{\tau}(a, b):=\int_{a}^{b} \int_{\mu}^{a} \eta^{\prime \prime}(\mu-\sigma) \sqrt{\phi^{\prime}(\sigma)} d \sigma \sqrt{\phi^{\prime}(\mu)} d \mu .
$$


Note that $\eta^{\prime \prime}(x) \leq C / \tau$ in $[-\tau, \tau]$ for a given constant, so that

$$
\begin{aligned}
& \left|A_{5}+A_{6}\right| \\
= & c(h) E \int_{Q \times \mathbb{R}^{d} \times(0,1)^{2}}\left|u_{2}(t, x, \epsilon)-u_{1}(t, y, \delta)\right|^{2} \varphi \rho_{m}(x-y) \eta^{\prime \prime}\left(u_{2}(t, x, \epsilon)-u_{1}(t, y, \delta)\right) d x d t d y \\
& +c(h) E \int_{Q \times \mathbb{R}^{d} \times(0,1)^{2}}\left|\omega_{h}(\|x-y\|)\right|^{2} \varphi \rho_{m}(x-y) \eta^{\prime \prime}\left(u_{2}(t, x, \epsilon)-u_{1}(t, y, \delta)\right) d x d t d y \\
\leq & c(h) \tau \int_{Q \times \mathbb{R}^{d}} \varphi \rho_{m}(x-y) d x d t d y+\frac{c(h)}{\tau} \int_{Q \times \mathbb{R}^{d}}\left|\omega_{h}(\|x-y\|)\right|^{2} \varphi \rho_{m}(x-y) d x d t d y \\
\leq & c(h) \tau \int_{Q} \varphi d x d t+\frac{c(h)\left|\omega_{h}\left(\frac{1}{m}\right)\right|^{2}}{\tau} \int_{Q} \varphi d x d t .
\end{aligned}
$$

Moreover,

$$
\begin{aligned}
&\left|A_{7}\right|=\mid E \int_{Q \times \mathbb{R}^{d} \times(0,1)^{2}} \varphi\left(F^{\eta}\left(u_{2}(t, x, \epsilon), u_{1}(t, y, \delta)\right)-\right.\left.F^{\eta}\left(u_{1}(t, y, \delta), u_{2}(t, x, \epsilon)\right)\right) \\
& \times \nabla \rho_{m}(x-y) d \epsilon d \delta d y d x d t \mid \\
& \leq C \tau \int_{Q \times \mathbb{R}^{d}} \varphi\left|\nabla \rho_{m}(x-y)\right| d y d x d t \leq \tau m C \int_{Q} \varphi d x d t
\end{aligned}
$$

- First situation: $h(x, u)=h(u)$. Then, $\omega_{h}=0$ and, $m$ being fixed, $\lim _{\tau \rightarrow 0} A_{4}+A_{5}+A_{6}+A_{7}=0$. Moreover,

$$
\begin{aligned}
& A_{8}+A_{9}+A_{10} \\
& =2 E \int_{Q \times \mathbb{R}^{d} \times(0,1)^{2}}\left[I_{\tau}\left(u_{1}(t, y, \delta), u_{2}(t, x, \epsilon)\right)+\phi^{\eta}\left(u_{2}(t, x, \epsilon), u_{1}(t, y, \delta)\right)\right] \\
& \times \nabla \varphi \nabla \rho_{m}(x-y) d \epsilon d \delta d y d x d t \\
& +E \int_{Q \times \mathbb{R}^{d} \times(0,1)^{2}}\left[2 I_{\tau}\left(u_{1}(t, y, \delta), u_{2}(t, x, \epsilon)\right)+\phi^{\eta}\left(u_{2}(t, x, \epsilon), u_{1}(t, y, \delta)\right)\right. \\
& \left.+\phi^{\eta}\left(u_{1}(t, y, \delta), u_{2}(t, x, \epsilon)\right)\right] \varphi \Delta \rho_{m}(x-y) d \epsilon d \delta d y d x d t .
\end{aligned}
$$

Note that, thanks to Lemma 2-(6), each integrand goes to 0 with $\tau$ and is bounded, respectively by

$$
\begin{array}{ll} 
& c\left(\phi^{\prime}\right)\left|u_{2}(t, x, \epsilon)-u_{1}(t, y, \delta)\right|\left|\nabla \varphi \nabla \rho_{m}(x-y)\right| \\
\text { and } \quad & c\left(\phi^{\prime}\right)\left|u_{2}(t, x, \epsilon)-u_{1}(t, y, \delta)\right|\left|\varphi \Delta \rho_{m}(x-y)\right| .
\end{array}
$$

Thus, one concludes that $\lim _{\tau \rightarrow 0} A_{8}+A_{9}+A_{10}=0$ and one can pass to the limit over $m$.

- Second situation: assume that $\phi=0$ or linear and that there exists $\theta \in$ $(0,1)$ such that $\frac{\omega_{h}(r)^{1+\theta}}{r} \rightarrow_{r \rightarrow 0} 0$ (this is the case for example if $\omega_{h}(r)=|r|^{\beta}$ for a given $\beta>1 / 2$ by setting $1>\theta>(1-\beta) / \beta)$.

Then, $A_{8}+A_{9}+A_{10}=0$ and by setting $\tau=\omega_{h}(1 / m)^{1+\theta}$, one has

$$
\left.\left|A_{4}+A_{5}+A_{6}+A_{7}+A_{8}+A_{9}+A_{10}\right| \leq C\left[\tau+\frac{\omega_{h}(1 / m)^{2}}{\tau}\right]+\tau m\right],
$$


and one concludes that $\lim _{m} A_{4}+A_{5}+A_{6}+A_{7}+A_{8}+A_{9}+A_{10}=0$.

- Last situation: assume the same for $h$, that $\phi$ is not linear and that $t \mapsto \sqrt{\phi^{\prime}(t)}$ has a modulus of continuity $\omega_{\phi}$ such that $\frac{\omega_{\phi}\left[\omega_{h}(r)^{1+\theta}\right]}{r} \rightarrow_{r \rightarrow 0} 0$ (this is the case for example if $\left.\omega_{\phi}(r)=C|r|\right)$.

By using the classical form of the mollifier sequence $\rho_{m}(x)=\mathrm{cm}^{d} \rho(m\|x\|)$ with $\rho(t)=e^{\frac{1}{t^{2}-1}} 1_{\{|t|<1\}}$, one gets that

$\nabla \rho_{m}(x)=m \rho_{m}(x) \frac{-2 m\|x\|}{\left(m^{2}\|x\|^{2}-1\right)^{2}} \frac{x}{\|x\|}$ and $\Delta \rho_{m}(x)=m^{2} \rho_{m}(x) \frac{P(m\|x\|)}{\left(m^{2}\|x\|^{2}-1\right)^{4}}$,

where $P(t)=(8-2 d) t^{4}+4(d-1) t^{2}-2 d$. Note that there exists $a \in(0,1)$ such that $P(t) \leq 0$ in $[0, a]$ and $P(t) \geq 0$ in $[a, 1]$, so that with (5) (see Lemma 2), $A_{8}+A_{9}+A_{10} \leq B$ where

$$
\begin{array}{r}
B:=2 E \int_{Q \times \mathbb{R}^{d} \times(0,1)^{2}}\left[I_{\tau}\left(u_{1}(t, y, \delta), u_{2}(t, x, \epsilon)\right)+\phi^{\eta}\left(u_{2}(t, x, \epsilon), u_{1}(t, y, \delta)\right)\right] \\
\times \nabla \varphi \nabla \rho_{m}(x-y) d \epsilon d \delta d y d x d t \\
+E \int_{\left[Q \times \mathbb{R}^{d} \times(0,1)^{2}\right] \cap\{m\|x-y\| \in[a, 1]\}}\left[\begin{array}{c}
{\left[2 I_{\tau}\left(u_{1}(t, y, \delta), u_{2}(t, x, \epsilon)\right)\right.} \\
\left.+\phi^{\eta}\left(u_{1}(t, y, \delta), u_{2}(t, x, \epsilon)\right)\right] \varphi \Delta \rho_{m}(x-y) d \epsilon d \delta d y d x d t .
\end{array}\right.
\end{array}
$$

Then, thanks to Lemma 2-(7), one has

$$
\begin{aligned}
|B| \leq & C \tau \int_{Q \times \mathbb{R}^{d}}\left|\nabla \varphi \nabla \rho_{m}(x-y)\right| d y d x d t \\
& +C \omega_{\phi}(\tau)^{2} E \int_{Q \times \mathbb{R}^{d} \times(0,1)^{2}}\left|u_{2}(t, x, \epsilon)-u_{1}(t, y, \delta)\right|\left[\left|\nabla \varphi \nabla \rho_{m}(x-y)\right|\right. \\
\leq & C m \tau+C m \omega_{\phi}(\tau)^{2} \sqrt{E \int_{Q \times \mathbb{R}^{d} \times(0,1)^{2}}\left|u_{2}(t, x, \epsilon)-u_{1}(t, y, \delta)\right|^{2} \rho_{m}(x-y) d \epsilon d \delta d y d x d t} \\
& +C m^{2} \omega_{\phi}(\tau)^{2} \sqrt{E \int_{Q \times \mathbb{R}^{d} \times(0,1)^{2}} \varphi \rho_{Q \times \mathbb{R}^{d}} \rho_{m}(x-y) \mid \frac{4 m^{2}\|x-y\|^{2}|\nabla \varphi|^{2}}{\left(m^{2}\|x-y\|^{2}-1\right)^{4}} d y d x d t} \\
& \times \sqrt{\int_{Q \times \mathbb{R}^{d}} \rho_{m}(x-y) \mid \frac{[\varphi P(m \| x-y)}{\left(m^{2}\|x-y\|^{2}-1\right)^{8}} 1_{\{m\|x-y\| \in[a, 1]\}} d y d x d t} \\
\leq & C\left[m \tau+m^{2} \omega_{\phi}(\tau)^{2}\right] .
\end{aligned}
$$

With the configuration of the previous situation, setting $\tau=\omega_{h}(1 / m)^{1+\theta}$ and $|B| \leq C m \tau+C\left(m \omega_{\phi}\left(\omega_{h}(1 / m)^{1+\theta}\right)^{2}\right.$. 
Then, with the assumption on the modulus $\omega_{\phi}, B$ converges to 0 when $m$ goes to $+\infty$.

Finally, whatever the situation, passing to the limit with respect to $m$, the following Kato inequality holds, for any $\varphi \in \mathcal{D}^{+}\left(\left[0, T\left[\times \mathbb{R}^{d}\right)\right.\right.$,

$$
\begin{aligned}
0 \leq & \int_{\mathbb{R}^{d}}\left|u_{0,2}-u_{0,1}\right| \varphi(0, x) d x \\
& +E \int_{Q \times(0,1)^{2}}\left(\left|u_{2}(\epsilon, \cdot)-u_{1}(\delta, \cdot)\right| \partial_{t} \varphi+\left|g\left(\cdot, u_{2}(\epsilon, \cdot)\right)-g\left(\cdot, u_{1}(\delta, \cdot)\right)\right| \varphi\right) d \delta d \epsilon d x d t \\
& +E \int_{Q \times(0,1)^{2}}\left(\left|\phi\left(u_{2}(\epsilon, \cdot)\right)-\phi\left(u_{1}(\delta, \cdot)\right)\right| \Delta \varphi-F\left(u_{2}(\epsilon, \cdot), u_{1}(\delta, \cdot)\right) \nabla \varphi\right) d \delta d \epsilon d x d t
\end{aligned}
$$

or, similarly, for any $\varphi \in D^{+}\left(\left[0, T\left[, H^{1}\left(\mathbb{R}^{d}\right)\right)\right.\right.$,

$$
\begin{aligned}
0 \leq & \int_{\mathbb{R}^{d}}\left|u_{0,2}-u_{0,1}\right| \varphi(0, x) d x \\
& +E \int_{Q \times(0,1)^{2}}\left(\left|u_{2}-u_{1}\right| \partial_{t} \varphi+\left|g\left(\cdot, u_{2}\right)-g\left(\cdot, u_{1}\right)\right| \varphi\right) d \delta d \epsilon d x d t \\
& -E \int_{Q}\left(\nabla \int_{(0,1)^{2}}\left|\phi\left(u_{2}\right)-\phi\left(u_{1}\right)\right| d \delta d \epsilon+\int_{(0,1)^{2}} F\left(u_{2}, u_{1}\right) d \delta d \epsilon\right) \nabla \varphi d x d t
\end{aligned}
$$

Following the idea of [4], denote by $\varphi(t, x)=\gamma(t) \alpha(x)$ where $\gamma \in \mathcal{D}^{+}([0, T[)$, $\alpha$ is the function defined by $\alpha(x)=\min \left(1, \frac{R^{a}}{|x|^{a}}\right)$ where $R \geq 1$ and $a=d / 2+\epsilon$, $\epsilon>0$ in order to have $\alpha$ in $L^{2}\left(\mathbb{R}^{d}\right)$.

Thus, $\nabla \alpha(x)=-a R^{a}|x|^{-a-1} \frac{x}{|x|} 1_{\{|x|>R\}}=-a \frac{\alpha(x)}{|x|} \frac{x}{|x|} 1_{\{|x|>R\}} \in L^{2}\left(\mathbb{R}^{d}\right)^{d}$; and, in the set $\{|x|>R\}$, one has that $\Delta \alpha(x)=a(2+2 \epsilon-a) \frac{\alpha(x)}{|x|^{2}}$ is in $L^{2}(\{|x|>R\})$. Thus,

$$
\begin{aligned}
0 \leq & \int_{\mathbb{R}^{d}}\left|u_{0,2}-u_{0,1}\right| \gamma(0) \alpha(x) d x \\
& +E \int_{Q \times(0,1)^{2}}\left[\left|u_{2}-u_{1}\right| \gamma^{\prime}(t)+\left|g\left(\cdot, u_{2}\right)-g\left(\cdot, u_{1}\right)\right| \gamma(t)\right] \alpha(x) d \delta d \epsilon d x d t \\
& +E \int_{0}^{T} \int_{\{|x>R|\} \times(0,1)^{2}}\left(\left|\phi\left(u_{2}\right)-\phi\left(u_{1}\right)\right| \Delta \alpha(x)-F\left(u_{2}, u_{1}\right) \nabla \alpha(x)\right) \gamma(t) d \delta d \epsilon d x d t \\
& -E \int_{0}^{T} \gamma(t) \int_{\partial\{|x|>R\} \times(0,1)^{2}}\left|\phi\left(u_{2}\right)-\phi\left(u_{1}\right)\right| \nabla \alpha \cdot \vec{n} d \delta d \epsilon d \sigma d t .
\end{aligned}
$$

Since $\nabla \alpha(x) \cdot \vec{n}=-\frac{a}{R} x \cdot \vec{n}=\frac{a}{R}>0$ on $\partial\{|x|>R\}$, this yields

$$
\begin{aligned}
0 \leq & \int_{\mathbb{R}^{d}}\left|u_{0,2}-u_{0,1}\right| \gamma(0) \alpha(x) d x \\
& +E \int_{Q \times(0,1)^{2}}\left[\left|u_{2}-u_{1}\right| \gamma^{\prime}(t)+\left|g\left(\cdot, u_{2}\right)-g\left(\cdot, u_{1}\right)\right| \gamma(t)\right] \alpha(x) d \delta d \epsilon d x d t \\
& +E \int_{0}^{T} \int_{\{|x>R|\} \times(0,1)^{2}} \alpha\left(\frac{a(2+2 \epsilon-a)}{|x|^{2}}\left|\phi\left(u_{2}\right)-\phi\left(u_{1}\right)\right|+\frac{a}{|x|} F\left(u_{2}, u_{1}\right) \cdot \frac{x}{|x|}\right) d \delta d \epsilon d x d t,
\end{aligned}
$$


and as $|x|>R$ in the last integral,

$$
\begin{aligned}
0 \leq & \int_{\mathbb{R}^{d}}\left|u_{0,2}-u_{0,1}\right| \gamma(0) \alpha(x) d x \\
& +E \int_{Q \times(0,1)^{2}}\left[\left|u_{2}-u_{1}\right| \gamma^{\prime}(t)+\left|g\left(\cdot, u_{2}\right)-g\left(\cdot, u_{1}\right)\right| \gamma(t)\right] \alpha(x) d \delta d \epsilon d x d t \\
& +C(d) \frac{R+1}{R^{2}} E \int_{0}^{T} \int_{\{|x>1|\} \times(0,1)^{2}} \alpha(x) \gamma(t)\left(\left|\phi\left(u_{2}\right)-\phi\left(u_{1}\right)\right|+\left|F\left(u_{2}, u_{1}\right)\right|\right) d \delta d \epsilon d x d t
\end{aligned}
$$

Using now that $R \geq 1$ and the Lipschitz properties of $\phi, \vec{f}$ and $g$,

$$
\begin{aligned}
0 \leq & \int_{\mathbb{R}^{d}}\left|u_{0,2}-u_{0,1}\right| \gamma(0) \alpha(x) d x \\
& +E \int_{Q \times(0,1)^{2}}\left|u_{2}-u_{1}\right| \alpha(x)\left[\gamma^{\prime}(t)+C(d, \phi, \vec{f}, g) \gamma(t)\right] d \delta d \epsilon d x d t
\end{aligned}
$$

Assume now, by an approximation argument, that $\gamma(t)=e^{-c t} \min \left(1, n(T-t)^{+}\right)$ where $c=C(d, \phi, \vec{f}, g)+1$, then

$$
\begin{aligned}
& n \int_{T-1 / n}^{T} E \int_{\mathbb{R}^{d} \times(0,1)^{2}}\left|u_{2}-u_{1}\right| \alpha(x) e^{-c t} d \delta d \epsilon d x d t \\
& +E \int_{Q \times(0,1)^{2}}\left|u_{2}-u_{1}\right| \alpha(x) e^{-c t} d \delta d \epsilon d x \min \left(1, n(T-t)^{+}\right) d t \\
\leq & \int_{\mathbb{R}^{d}}\left|u_{0,2}-u_{0,1}\right| \alpha(x) d x
\end{aligned}
$$

Thus, if one assumes that $u_{0,2}=u_{0,1}$, passing to the limit over $n$ yields,

$$
E \int_{Q \times(0,1)^{2}}\left|u_{2}-u_{1}\right| \alpha(x) e^{-c t} d \delta d \epsilon d x d t \leq 0 .
$$

This means on the one hand that $u_{1}$ and $u_{2}$ are the same functions, but also on the other hand that they are not functions of the additional variables $\epsilon$ and $\delta$ respectively. Thus, one is able to conclude that the whole sequence of viscous approximation converges, weakly in $L^{2}(\Omega \times Q)$ and strongly in $L^{p}(\Omega \times$ $\left.(0, T), L_{l o c}^{p}\left(\mathbb{R}^{d}\right)\right)$ for any $p<2$ to a weak entropy solution $u$ in the sense of our definition.

Then, back to (4), one gets by passing to the limit over $n$,

$$
\begin{aligned}
& \quad \liminf _{n} n \int_{T-1 / n}^{T} E \int_{\mathbb{R}^{d}}\left|u_{2}-u_{1}\right| \alpha(x) e^{-c t} d x d t \\
& +E \int_{Q}\left|u_{2}-u_{1}\right| \alpha(x) e^{-c t} d x d t \\
& \leq \int_{\mathbb{R}^{d}}\left|u_{0,2}-u_{0,1}\right| \alpha(x) d x
\end{aligned}
$$


Thanks to Remark $1, t \mapsto u_{2}-u_{1}$ is weakly continuous with values in $L^{2}\left(\Omega \times \mathbb{R}^{d}\right)$ and since $u \in L^{2}\left(\Omega \times \mathbb{R}^{d}\right) \mapsto E \int_{\mathbb{R}^{d}}|u| \alpha d x$ is a non-negative convex continuous function, it is l.s.c. for the weak topology and

$$
\begin{aligned}
& E \int_{\mathbb{R}^{d}}\left|u_{2}-u_{1}\right|(T) \alpha(x) d x+E \int_{Q}\left|u_{2}-u_{1}\right|(t) \alpha(x) e^{c(T-t)} d x d t \\
\leq & e^{c T} \int_{\mathbb{R}^{d}}\left|u_{0,2}-u_{0,1}\right| \alpha(x) d x .
\end{aligned}
$$

Since the time $T$ is arbitrary, this last assertion closes the proof of the existence of a solution, limit of the viscous approximation and the stability of such solutions in $L^{1}(\mathbb{R}, \alpha d x)$. After the proof of the uniqueness of the solution in the sense of Definition 1 (see next section) this will prove the first part of the theorem.

Assume now that the initial conditions and $g(\cdot, 0)$ are elements of $L^{1}\left(\mathbb{R}^{d}\right)$ and also $h(\cdot, 0)=0$. Thus, thanks to Remark 2, the corresponding solutions are in $L^{\infty}\left(0, T, L^{1}\left(\Omega \times \mathbb{R}^{d}\right)\right)$.

Then, the above estimate yields

$$
\begin{aligned}
& E \int_{\mathbb{R}^{d}}\left|u_{2}-u_{1}\right|(T) \min \left(1, \frac{R^{a}}{|x|^{a}}\right) d x \\
\leq & e^{c T} \int_{\mathbb{R}^{d}}\left|u_{0,2}-u_{0,1}\right| \min \left(1, \frac{R^{a}}{|x|^{a}}\right) d x \leq e^{c T} \int_{\mathbb{R}^{d}}\left|u_{0,2}-u_{0,1}\right| d x .
\end{aligned}
$$

Thus, by Beppo Levi's theorem, one concludes that $\left(u_{2}-u_{1}\right)(t)$, a priori element of $L^{2}\left(\Omega \times \mathbb{R}^{d}\right)$, is an element of $L^{1}\left(\Omega \times \mathbb{R}^{d}\right)$ with the information that

$$
E \int_{\mathbb{R}^{d}}\left|u_{2}-u_{1}\right|(T) d x \leq e^{c T} \int_{\mathbb{R}^{d}}\left|u_{0,2}-u_{0,1}\right| d x .
$$

\section{Uniqueness of the solution}

Our aim is to prove, as in [1], that any solution in the sense of our definition is unique by proving that it is equal to the solution obtained by viscous approximation. The method used to prove this result is exactly the same as the one proposed in the section dedicated to the result of existence, considering a solution $u$ (i.e. $\epsilon=0)$ and $u^{\delta}$. Coming back to the proofs, the only difference lies in the terms $I_{1}$ and $I_{3}$ where one has to set $\epsilon=0$. In the other terms, the proofs are the same since we used intentionally the generalized chain-rule (Lemma 3) instead of the classical one for Sobolev-functions since $u$ is in general not a Sobolev-function, but $G(u)$ is.

This remark allows us to prove the theorem. 


\section{Technical lemmata}

Lemma 1 For any Lipschitz-continuous function $g: \mathbb{R} \rightarrow \mathbb{R}$ and any $\eta \in \mathcal{E}$,

$$
\forall x, k \in \mathbb{R}, \quad\left|g^{\eta}(x, k)-s g n_{0}(x-k)[g(x)-g(k)]\right| \leq \operatorname{lip}(g) \tau .
$$

Proof. This comes from the remark that $\forall x, k \in \mathbb{R}$,

$\left|g^{\eta}(x, k)-\operatorname{sgn}_{0}(x-k)[g(x)-g(k)]\right|=\left|\int_{k}^{x}\left[\eta^{\prime}(\sigma-k)-\operatorname{sgn}_{0}(\sigma-k)\right] g^{\prime}(\sigma) d \sigma\right|$.

Lemma 2 Set, for any $a, b$ :

$$
I_{\tau}(a, b):=\int_{a}^{b} \int_{\mu}^{a} \eta^{\prime \prime}(\mu-\sigma) \sqrt{\phi^{\prime}(\sigma)} d \sigma \sqrt{\phi^{\prime}(\mu)} d \mu .
$$

Then, $I_{\tau}(a, b)=I_{\tau}(b, a)$,

$$
\begin{aligned}
& 0 \leq 2 I_{\tau}(a, b)+\phi^{\eta}(a, b)+\phi^{\eta}(b, a)=\frac{1}{2} \int_{a}^{b} \int_{a}^{b} \eta^{\prime \prime}(\mu-\sigma)\left[\sqrt{\phi^{\prime}(\sigma)}-\sqrt{\phi^{\prime}(\mu)}\right]^{2} d \mu d \sigma \\
& \leq 2\left\|\phi^{\prime}\right\|_{\infty}|b-a| \\
& \text { and } \quad 3\left\|\phi^{\prime}\right\|_{\infty}|b-a| \geq\left|I_{\tau}(a, b)+\phi^{\eta}(b, a)\right|+\left|I_{\tau}(a, b)+\phi^{\eta}(a, b)\right| \rightarrow_{\tau \rightarrow 0} 0
\end{aligned}
$$

Moreover, if $\sqrt{\phi^{\prime}}$ admits a modulus of continuity $\omega_{\phi}$, then

$$
\begin{gathered}
2 I_{\tau}(a, b)+\phi^{\eta}(a, b)+\phi^{\eta}(b, a) \leq w_{\phi}(\tau)^{2}|b-a| \\
\max \left[\left|I_{\tau}(a, b)+\phi^{\eta}(b, a)\right|,\left|I_{\tau}(a, b)+\phi^{\eta}(a, b)\right|\right] \\
\leq \frac{1}{2} w_{\phi}(\tau)^{2}|b-a|+\left\|\phi^{\prime}\right\|_{\infty} \min (\tau,|b-a|) .
\end{gathered}
$$

Proof. Note that, by Fubini's theorem,

$$
\begin{aligned}
I_{\tau}(a, b) & =-\int_{a}^{b} \int_{a}^{\mu} \eta^{\prime \prime}(\mu-\sigma) \sqrt{\phi^{\prime}(\sigma)} \sqrt{\phi^{\prime}(\mu)} d \sigma d \mu \\
& =-\int_{a}^{b} \int_{\sigma}^{b} \eta^{\prime \prime}(\sigma-\mu) \sqrt{\phi^{\prime}(\sigma)} \sqrt{\phi^{\prime}(\mu)} d \sigma d \mu
\end{aligned}
$$

and since $\eta^{\prime \prime}$ is even, one gets that $I_{\tau}(a, b)=I_{\tau}(b, a)$ since the above remark yields

$$
I_{\tau}(a, b)=-\frac{1}{2} \int_{a}^{b} \int_{a}^{b} \eta^{\prime \prime}(\mu-\sigma) \sqrt{\phi^{\prime}(\sigma)} \sqrt{\phi^{\prime}(\mu)} d \sigma d \mu .
$$


As $\eta^{\prime}$ is odd and $\eta(r) \leq|r|$ when $|r| \leq \tau$, we get

$$
\begin{aligned}
& 2 I_{\tau}(a, b)+\phi^{\eta}(a, b)+\phi^{\eta}(b, a) \\
= & \int_{a}^{b}\left[\left[\eta^{\prime}(\mu-a)-\eta^{\prime}(\mu-b)\right] \phi^{\prime}(\mu)-\int_{a}^{b} \eta^{\prime \prime}(\mu-\sigma) \sqrt{\phi^{\prime}(\sigma)} \sqrt{\phi^{\prime}(\mu)} d \sigma\right) d \mu \\
= & \int_{a}^{b} \int_{a}^{b} \eta^{\prime \prime}(\sigma-\mu)\left[\sqrt{\phi^{\prime}(\mu)}-\sqrt{\phi^{\prime}(\sigma)}\right] \sqrt{\phi^{\prime}(\mu)} d \sigma d \mu \\
= & \int_{a}^{b} \int_{a}^{b} \eta^{\prime \prime}(\mu-\sigma)\left[\sqrt{\phi^{\prime}(\sigma)}-\sqrt{\phi^{\prime}(\mu)}\right] \sqrt{\phi^{\prime}(\sigma)} d \mu d \sigma \\
= & -\int_{a}^{b} \int_{a}^{b} \eta^{\prime \prime}(\sigma-\mu)\left[\sqrt{\phi^{\prime}(\mu)}-\sqrt{\phi^{\prime}(\sigma)}\right] \sqrt{\phi^{\prime}(\sigma)} d \mu d \sigma \\
= & \frac{1}{2} \int_{a}^{b} \int_{a}^{b} \eta^{\prime \prime}(\mu-\sigma)\left[\sqrt{\phi^{\prime}(\sigma)}-\sqrt{\phi^{\prime}(\mu)}\right]^{2} d \mu d \sigma \leq\left\|\phi^{\prime}\right\|_{\infty}|b-a| .
\end{aligned}
$$

Therefore,

$$
\begin{aligned}
& \sup \left[I_{\tau}(a, b)+\phi^{\eta}(b, a), I_{\tau}(a, b)+\phi^{\eta}(a, b)\right] \\
\leq & \frac{1}{4} \int_{a}^{b} \int_{a}^{b} \eta^{\prime \prime}(\mu-\sigma)\left[\sqrt{\phi^{\prime}(\sigma)}-\sqrt{\phi^{\prime}(\mu)}\right]^{2} d \mu d \sigma+\frac{1}{2} \int_{a}^{b}\left|\eta^{\prime}(\mu-a)+\eta^{\prime}(\mu-b)\right| \phi^{\prime}(\sigma) d \sigma \\
\leq & \frac{1}{4} \int_{a}^{b} \int_{a}^{b} \eta^{\prime \prime}(\mu-\sigma)\left[\sqrt{\phi^{\prime}(\sigma)}-\sqrt{\phi^{\prime}(\mu)}\right]^{2} d \mu d \sigma+\left\|\phi^{\prime}\right\|_{\infty} \min (\tau,|b-a|) \\
\leq & 2\left\|\phi^{\prime}\right\|_{\infty}|b-a|+\left\|\phi^{\prime}\right\|_{\infty} \min (\tau,|b-a|) .
\end{aligned}
$$

Finally, if one assumes that $b>a(a>b$ is similar $)$, then

$$
\begin{aligned}
& \int_{a}^{b} \int_{a}^{b} \eta^{\prime \prime}(\mu-\sigma)\left[\sqrt{\phi^{\prime}(\sigma)}-\sqrt{\phi^{\prime}(\mu)}\right]^{2} d \mu d \sigma \\
\leq & \int_{a}^{b} \int_{-\tau}^{\tau} \eta^{\prime \prime}(\alpha)\left|\sqrt{\phi^{\prime}(\mu)}-\sqrt{\phi^{\prime}(\mu+\alpha)}\right|^{2} d \alpha d \mu \\
= & \int_{-\tau}^{\tau} \eta^{\prime \prime}(\alpha) \int_{a}^{b}\left|\sqrt{\phi^{\prime}(\mu)}-\sqrt{\phi^{\prime}(\mu+\alpha)}\right|^{2} d \mu d \alpha .
\end{aligned}
$$

In particular, since $\lim _{\alpha \rightarrow 0} \int_{a}^{b}\left|\sqrt{\phi^{\prime}(\mu-\alpha)}-\sqrt{\phi^{\prime}(\mu)}\right|^{2} d \mu=0$ by continuity of the translations in $L^{2}$, one gets the convergence to 0 claimed in the lemma.

Lemma 3 (A generalized chain rule) Consider $\mathcal{O} \subset \mathbb{R}^{d}$ a domain with a Lipschitz boundary (if there is a boundary), $u \in L^{2}(\mathcal{O}), f \in L^{\infty}(\mathbb{R}), G$ a Lipschitz-continuous function with $G(0)=0$ and assume that $G(u) \in H^{1}(\mathcal{O})$. Then $v=\int_{b}^{u} f(s) G^{\prime}(s) d s \in H^{1}(\mathcal{O})$ where $b \in \mathbb{R}$ if $\mathcal{O}$ has a finite measure and $b=0$ else, and, a.e.

$$
\nabla \int_{b}^{u} f(s) G^{\prime}(s) d s=f(u) \nabla G(u) .
$$


Proof. First, assume that $f$ is a continuous function and that $G$ is a nondecreasing function.

Set $G_{\epsilon}: x \mapsto G_{\epsilon}(x)=G(x)+\epsilon x$. $G_{\epsilon}^{-1}$ is well-defined on $\mathbb{R}$ and it is a Lipschitzcontinuous function. Then, the classical chain rule yields

$f \circ G_{\epsilon}^{-1}(G(u)) \nabla G(u)=\nabla \int_{G(b)}^{G(u)} f \circ G_{\epsilon}^{-1}(s) d s=\nabla \int_{b}^{u} f \circ G_{\epsilon}^{-1}(G(s)) G^{\prime}(s) d s$.

Note that $G_{\epsilon}(0)=0, G_{\epsilon}(x)<0$ if $x<0$ and $G_{\epsilon}(x)>0$ if $x>0$.

If $x>0$, one has $x=G_{\epsilon}^{-1}\left(G_{\epsilon}(x)\right)>G_{\epsilon}^{-1}(G(x))$ and $x \geq \lim \sup _{\epsilon} G_{\epsilon}^{-1}(G(x))$. Our aim is to show that $G_{\epsilon}^{-1}(G(x)) \rightarrow x$. To this end we consider the two following possible cases:

- Assume that $x$ is such that: $\forall y \in \mathbb{R}, y<x \Rightarrow G(y)<G(x)$.

Then, for small $\epsilon \leq \epsilon_{y}, G(x)>G(y)+\epsilon y$ and $x>G_{\epsilon}^{-1}(G(x))>G_{\epsilon}^{-1}\left(G_{\epsilon}(y)\right)=$ $y$. Thus, $\liminf _{\epsilon} G_{\epsilon}^{-1}(G(x)) \geq y$, and at the limit when $y \rightarrow x^{-}$, one gets $G_{\epsilon}^{-1}(G(x)) \rightarrow x$.

- Assume now that there exists $0 \leq y<x$ such that $G(y)=G(x)$. If one denotes by $a(x)=\inf \{y \in[0, x], G(y)=G(x)\}=\min \{y \in[0, x], G(y)=G(x)\}$.

$G(a(x))=G(x)$ and, regarding the definition of $a(x)$ and the previous case, $G_{\epsilon}^{-1}(G(x))=G_{\epsilon}^{-1}(G(a(x))) \rightarrow a(x)$.

Conclusion: for any $x \geq 0, G_{\epsilon}^{-1}(G(x)) \rightarrow a(x)$.

Similarly, for any $x \leq 0, x \leq G_{\epsilon}^{-1}(G(x)) \rightarrow a(x)=\max \{y \in[x, 0], G(y)=$ $G(x)\}$ in that case.

Thus, for any real $x, G_{\epsilon}^{-1}(G(x)) \rightarrow a(x)=\operatorname{argmin}\{|y|, G(y)=G(x)\}$ with $\left|G_{\epsilon}^{-1}(G(x))\right| \leq|x|$.

Thus, since $f$ is a bounded continuous function,

$$
\int_{b}^{u} f o G_{\epsilon}^{-1}(G(s)) G^{\prime}(s) d s \rightarrow \int_{b}^{u} f(a(s)) G^{\prime}(s) d s=\int_{b}^{u} f(s) G^{\prime}(s) d s \quad \text { a.e. }
$$

since, if $a(s) \neq s$, then $G^{\prime}(s)=0$, unless for a countable number of points.

$f$ and $G^{\prime}$ are bounded function, so that the Lebesgue theorem yields the convergence of $\int_{b}^{u} f o G_{\epsilon}^{-1}(G(s)) G^{\prime}(s) d s$ to $\int_{b}^{u} f(s) G^{\prime}(s) d s$ in $L^{2}(\mathcal{O})$, thus in the sense of distributions, and then $\nabla \int_{b}^{u} f o G_{\epsilon}^{-1}(G(s)) G^{\prime}(s) d s$ converges to $\nabla \int_{b}^{u} f(s) G^{\prime}(s) d s$ in the sense of distributions.

On the other hand, $f_{o} G_{\epsilon}^{-1}(G(u)) \nabla G(u)$ converges to $f(a(u)) \nabla G(u)$ a.e. and $\left|f o G_{\epsilon}^{-1}(G(u)) \nabla G(u)\right| \leq c|\nabla G(u)|$, thus $f o G_{\epsilon}^{-1}(G(u)) \nabla G(u)$ converges to $f(a(u)) \nabla G(u)$ in $L^{2}(\mathcal{O})^{d}$.

Denote by $D=\{s \neq a(s)\}$. It is a countable set and, a.e.,

$$
\begin{aligned}
|[f(a(u))-f(u)] \nabla G(u)| & \leq|f(a(u))-f(u)||\nabla G(u)| 1_{\{u \in D\}} \\
& \leq|f(a(u))-f(u)||\nabla G(u)| 1_{\{G(u) \in G(D)\}}=0
\end{aligned}
$$


thanks to Saks lemma and since $G(D)$ is at most countable.

The conclusion is then that, in $L^{2}(\mathcal{O})^{d}$,

$f(u) \nabla G(u) \leftarrow f o G_{\epsilon}^{-1}(G(u)) \nabla G(u)=\nabla \int_{b}^{u} f o G_{\epsilon}^{-1}(G(s)) G^{\prime}(s) d s \rightarrow \nabla \int_{b}^{u} f(s) G^{\prime}(s) d s$

and the result holds in that first case: a non-decreasing Lipschitz function $G$ and a bounded continuous function $f$.

Note that the same proof yields the result for a decreasing Lipschitz function $G$ and any pointwise limit of sequences of bounded continuous function $\left(f_{n}\right)$, uniformly bounded by a same constant. Thus, the result holds for the Baire class of uniformly bounded continuous function, i.e., the bounded Borel functions $f$. Now, since, in the Lebesgue class of $f \in L^{\infty}(\mathbb{R})$, there exists a Borel function $\bar{f}$, bounded by the same value $\|f\|_{L^{\infty}(\mathbb{R})}$, one has

$$
\bar{f}(u) \nabla G(u)=\nabla \int_{b}^{u} \bar{f}(s) G^{\prime}(s) d s=\nabla \int_{b}^{u} f(s) G^{\prime}(s) d s .
$$

If now $D$ denotes the negligible set where $f$ and $\bar{f}$ differs,

$$
|[\bar{f}(u)-f(u)] \nabla G(u)| \leq|\bar{f}(u)-f(u)||\nabla G(u)| 1_{\{G(u) \in G(D)\}}=0 \quad \text { a.e. }
$$

since $G(D)$ is negligible ( $D$ is negligible and $G$ Lipschitz, non-decreasing) and by using Saks Lemma.

To finish the proof, just remind that any Lipschitz function is the difference of two Lipschitz non-decreasing functions.

Lemma 4 The weak solution $u^{\epsilon}$ to Problem $\left(P_{\epsilon}\right)$ satisfies the following estimates:

$$
\begin{aligned}
& \sup _{t}\left\|u^{\epsilon}\right\|_{L^{2}\left(\Omega \times \mathbb{R}^{d}\right)}^{2}(t)+\epsilon\left\|\nabla u^{\epsilon}\right\|_{L^{2}(\Omega \times Q)}^{2}+\left\|\nabla G\left(u^{\epsilon}\right)\right\|_{L^{2}(\Omega \times Q}^{2} \leq C . \\
& \text { If, moreover, } u_{0}, g(\cdot, 0) \in L^{1}\left(\mathbb{R}^{d}\right) \text { and } h(\cdot, 0)=0, \\
& \sup _{t} E \int_{\mathbb{R}^{d}} \tilde{\eta}\left(u^{\epsilon}(t)\right) d x \leq e^{c(h, g) T}\left[\int_{\mathbb{R}^{d}} \tilde{\eta}\left(u_{0}^{\epsilon}\right) d x+T c(g)\left[\tau+\|g(x, 0)\|_{L^{1}\left(\mathbb{R}^{d}\right)}\right]\right.
\end{aligned}
$$

where $\tilde{\eta}$ denotes the even convex function, defined for any positive $x$ by: $\tilde{\eta}(x)=$ $\frac{x^{2}}{2 \tau} 1_{\{\| x \mid<\tau\}}+\left(x-\frac{\tau}{2}\right) 1_{\{|| x \mid \geq \tau\}}$.

Proof. Denote by $\eta$ a non-negative convex-function, with $\eta^{\prime}$ Lipschitz-continuous, and assume that $|\eta(u)| \leq C|u|^{2}$ for a given constant $C$. Thanks to Itô's formula, for any $t$,

$$
\begin{aligned}
& \int_{\mathbb{R}^{d}} \eta\left(u^{\epsilon}(t)\right) d x+\int_{0}^{t} \int_{\mathbb{R}^{d}} \eta^{\prime \prime}\left(u^{\epsilon}\right)\left[\epsilon+\phi^{\prime}\left(u^{\epsilon}\right)\right]\left|\nabla u^{\epsilon}\right|^{2}+\eta^{\prime \prime}\left(u^{\epsilon}\right) \vec{f}\left(u^{\epsilon}\right) \nabla u^{\epsilon} d x d s \\
= & \int_{\mathbb{R}^{d}} \eta\left(u_{0}^{\epsilon}\right) d x+\int_{0}^{t} \int_{\mathbb{R}^{d}} h\left(x, u^{\epsilon}\right) \eta^{\prime}\left(u^{\epsilon}\right) d x d w(s) \\
& +\frac{1}{2} \int_{0}^{t} \int_{\mathbb{R}^{d}} h\left(x, u^{\epsilon}\right)^{2} \eta^{\prime \prime}\left(u^{\epsilon}\right) d x d s+\int_{0}^{t} \int_{\mathbb{R}^{d}} g\left(x, u^{\epsilon}\right) \eta^{\prime}\left(u^{\epsilon}\right) d x d s .
\end{aligned}
$$


Therefore,

$$
\begin{aligned}
E \int_{\mathbb{R}^{d}} \eta\left(u^{\epsilon}(t)\right) d x+E \int_{0}^{t} \int_{\mathbb{R}^{d}} \eta^{\prime \prime}\left(u^{\epsilon}\right) \epsilon\left|\nabla u^{\epsilon}\right|^{2}+ & \eta^{\prime \prime}\left(u^{\epsilon}\right)\left|\nabla G\left(u^{\epsilon}\right)\right|^{2} \\
& +\operatorname{div}\left[\int_{0}^{u^{\epsilon}} \eta^{\prime \prime}(\sigma) \vec{f}(\sigma) d \sigma\right] d x d s \\
\leq & \int_{\mathbb{R}^{d}} \eta\left(u_{0}^{\epsilon}\right) d x+c(h) E \int_{0}^{t} \int_{\mathbb{R}^{d}}\left(u^{\epsilon}\right)^{2} \eta^{\prime \prime}\left(u^{\epsilon}\right) d x d s+2 E \int_{0}^{t} \int_{\mathbb{R}^{d}} h(x, 0)^{2} \eta^{\prime \prime}\left(u^{\epsilon}\right) d x d s \\
& +c(g) E \int_{0}^{t} \int_{\mathbb{R}^{d}}\left(\left|u^{\epsilon}\right|+|g(x, 0)|\right)\left|\eta^{\prime}\left(u^{\epsilon}\right)\right| d x d s .
\end{aligned}
$$

Note that if $u \in H^{1}\left(\mathbb{R}^{d}\right)$, there exists a sequence $\left(u_{n}\right) \in D\left(\mathbb{R}^{d}\right)$ that converges to $u$ in $H^{1}\left(\mathbb{R}^{d}\right)$, so that

$$
\begin{aligned}
\int_{\mathbb{R}^{d}} \vec{f}(u) \nabla \eta^{\prime}(u) d x & =\lim _{n} \int_{\mathbb{R}^{d}} \vec{f}\left(u_{n}\right) \nabla \eta^{\prime}\left(u_{n}\right) d x=\lim _{n} \int_{\mathbb{R}^{d}} \vec{f}\left(u_{n}\right) \eta^{\prime \prime}\left(u_{n}\right) \nabla u_{n} d x \\
& =\lim _{n} \int_{\mathbb{R}^{d}} \operatorname{div}\left[\int_{0}^{u_{n}} \vec{f}(\sigma) \eta^{\prime \prime}(\sigma) d \sigma\right] d x=0 .
\end{aligned}
$$

Thus,

$$
\begin{aligned}
& E \int_{\mathbb{R}^{d}} \eta\left(u^{\epsilon}(t)\right) d x+E \int_{0}^{t} \int_{\mathbb{R}^{d}} \eta^{\prime \prime}\left(u^{\epsilon}\right) \epsilon\left|\nabla u^{\epsilon}\right|^{2}+\eta^{\prime \prime}\left(u^{\epsilon}\right)\left|\nabla G\left(u^{\epsilon}\right)\right|^{2} d x d s \\
\leq & \int_{\mathbb{R}^{d}} \eta\left(u_{0}^{\epsilon}\right) d x+c(h) E \int_{0}^{t} \int_{\mathbb{R}^{d}}\left(u^{\epsilon}\right)^{2} \eta^{\prime \prime}\left(u^{\epsilon}\right) d x d s+2 E \int_{0}^{t} \int_{\mathbb{R}^{d}} h(x, 0)^{2} \eta^{\prime \prime}\left(u^{\epsilon}\right) d x d s \\
& +c(g) E \int_{0}^{t} \int_{\mathbb{R}^{d}}\left(\left|u^{\epsilon}\right|+|g(x, 0)|\right)\left|\eta^{\prime}\left(u^{\epsilon}\right)\right| d x d s .
\end{aligned}
$$

Assume first that $\eta(x)=x^{2}$. Then, this yields (the constants $c(h)$ and $c(g)$ may change from one line to another)

$$
\begin{aligned}
& \left\|u^{\epsilon}\right\|_{L^{2}\left(\Omega \times \mathbb{R}^{d}\right)}^{2}(t)+\epsilon\left\|\nabla u^{\epsilon}\right\|_{L^{2}(\Omega \times Q}^{2}+\left\|\nabla G\left(u^{\epsilon}\right)\right\|_{L^{2}(\Omega \times Q)}^{2} \\
\leq & \left\|u_{0}^{\epsilon}\right\|_{L^{2}\left(\mathbb{R}^{d}\right)}^{2}+[c(h)+c(g)] \int_{0}^{t}\left\|u^{\epsilon}\right\|_{L^{2}\left(\Omega \times \mathbb{R}^{d}\right)}^{2}(s) d s \\
& +c(g) T\|g(x, 0)\|_{L^{2}\left(\mathbb{R}^{d}\right)}^{2}+4 T\|h(x, 0)\|_{L^{2}\left(\mathbb{R}^{d}\right)}^{2},
\end{aligned}
$$

and Gronwall's lemma implies that

$$
\sup _{t}\left\|u^{\epsilon}\right\|_{L^{2}\left(\Omega \times \mathbb{R}^{d}\right)}^{2}(t)+\epsilon\left\|\nabla u^{\epsilon}\right\|_{L^{2}(\Omega \times Q)}^{2}+\left\|\nabla G\left(u^{\epsilon}\right)\right\|_{L^{2}(\Omega \times Q}^{2} \leq C .
$$

Assume now that $u_{0}, g(\cdot, 0) \in L^{1}\left(\mathbb{R}^{d}\right)$ and that $h(\cdot, 0)=0$ and denote by $\eta=\tilde{\eta}$ the classical even and convex approximation of the absolute value function introduced in the lemma. Note that $0 \leq \tilde{\eta}(x) \leq \frac{4 x^{2}}{\tau},\left|x \tilde{\eta}^{\prime}(x)\right| \leq 2 \tilde{\eta}(x)+\tau$ and 
that $x^{2} \tilde{\eta}^{\prime \prime}(x) \leq \tilde{\eta}(x)$ for any $x$. Then,

$$
\begin{aligned}
E \int_{\mathbb{R}^{d}} \tilde{\eta}\left(u^{\epsilon}(t)\right) d x \leq & \int_{\mathbb{R}^{d}} \tilde{\eta}\left(u_{0}^{\epsilon}\right) d x+c(h, g) E \int_{0}^{t} \int_{\mathbb{R}^{d}} \tilde{\eta}\left(u^{\epsilon}\right) d x d s \\
& +T c(g)\left[\tau+\|g(x, 0)\|_{L^{1}\left(\mathbb{R}^{d}\right)}\right],
\end{aligned}
$$

and thanks to Gronwall's lemma,

$$
\sup _{t} E \int_{\mathbb{R}^{d}} \tilde{\eta}\left(u^{\epsilon}(t)\right) d x \leq e^{c(h, g) T}\left[\int_{\mathbb{R}^{d}} \tilde{\eta}\left(u_{0}^{\epsilon}\right) d x+T c(g)\left[\tau+\|g(x, 0)\|_{L^{1}\left(\mathbb{R}^{d}\right)}\right] .\right.
$$

Remark 2 Assume that $u_{0}, g(\cdot, 0) \in L^{1}\left(\mathbb{R}^{d}\right), h(\cdot, 0)=0$ and that $\left(u^{\epsilon}\right)$ converges weakly to a given $u$ in $L^{2}(\Omega \times Q)$. Since $\tilde{\eta}$ is a convex continuous function, one gets at the limit that:

$\int_{\Omega \times Q} \tilde{\eta}(u) d x d t \leq \liminf _{\epsilon} \int_{\Omega \times Q} \tilde{\eta}\left(u^{\epsilon}\right) d x d t \leq C\left[\int_{\mathbb{R}^{d}} \tilde{\eta}\left(u_{0}\right) d x+1\right] \leq C\left[\left\|u_{0}\right\|_{L^{1}\left(\mathbb{R}^{d}\right)}+1\right]$.

Since $\tilde{\eta}$ is monotone with respect to its parameter $\tau$, Beppo Levi's theorem yields $u \in L^{\infty}\left(0, T, L^{1}\left(\Omega \times \mathbb{R}^{d}\right)\right)$.

Acknowledgment. The authors would like to thank Boris Andreianov for his valuable suggestions.

\section{References}

[1] C. Bauzet, G. Vallet, P. Wittbold, The Cauchy problem for a conservation law with a multiplicative stochastic perturbation, Journal of Hyperbolic Differential Equations 09 (04) (2012) 661-709.

[2] G.-Q. Chen, K. H. Karlsen, Quasilinear anisotropic degenerate parabolic equations with time-space dependent diffusion coefficients, Commun. Pure Appl. Anal. 4 (2) (2005) 241-266. doi:10.3934/cpaa.2005.4.241.

URL http://dx.doi.org/10.3934/cpaa.2005.4.241

[3] J. Carrillo, Entropy solutions for nonlinear degenerate problems, Arch. Ration. Mech. Anal. 147 (4) (1999) 269-361.

[4] B. Andreianov, M. Maliki, A note on uniqueness of entropy solutions to degenerate parabolic equations in $\mathbb{R}^{N}$, NoDEA Nonlinear Differential Equations Appl. 17 (1) (2010) 109-118. doi:10.1007/s00030-009-0042-9.

URL http://dx.doi.org/10.1007/s00030-009-0042-9

[5] W. E, K. Khanin, A. Mazel, Y. Sinai, Invariant measures for Burgers equation with stochastic forcing, Ann. Math. (2) 151 (3) (2000) 877-960. 
[6] J. U. Kim, On a stochastic scalar conservation law, Indiana Univ. Math. J. 52 (1) (2003) 227-256.

[7] G. Vallet, P. Wittbold, On a stochastic first-order hyperbolic equation in a bounded domain, Infinite Dimensional Analysis, Quantum Probability 12 (4) (2009) 1-39.

[8] J. Feng, D. Nualart, Stochastic scalar conservation laws, J. Funct. Anal. 255 (2) (2008) 313-373.

[9] G.-Q. Chen, Q. Ding, K. H. Karlsen, On nonlinear stochastic balance laws, Arch. Ration. Mech. Anal. 204 (3) (2012) 707-743.

[10] A. Debussche, J. Vovelle, Scalar conservation laws with stochastic forcing, J. Funct. Anal. 259 (4) (2010) 1014-1042. doi:10.1016/j.jfa.2010.02.016. URL http://dx.doi.org/10.1016/j.jfa.2010.02.016

[11] C. Bauzet, G. Vallet, P. Wittbold, The Dirichlet problem for a conservation law with a multiplicative stochastic perturbation, To appear in J. Funct. Anal. dx.doi.org/10.1016/j.jfa.2013.06.022.

[12] G. Da Prato, J. Zabczyk, Stochastic equations in infinite dimensions, Vol. 44 of Encyclopedia of Mathematics and its Applications, Cambridge University Press, Cambridge, 1992.

[13] C. Prévôt, M. Röckner, A concise course on stochastic partial differential equations, Vol. 1905 of Lecture Notes in Mathematics, Springer, Berlin, 2007.

[14] G. Vallet, Stochastic perturbation of nonlinear degenerate parabolic problems, Differential and Integral Equations 21 (11-12) (2008) 1055-1082.

[15] D. Fellah, E. Pardoux, Une formule d'Itô dans des espaces de Banach et application, in: Stochastic analysis and related topics (Silivri, 1990), Vol. 31 of Progr. Probab., Birkhäuser Boston, Boston, MA, 1992, pp. 197-209.

[16] R. Temam, Navier-Stokes equations, revised Edition, Vol. 2 of Studies in Mathematics and its Applications, North-Holland Publishing Co., Amsterdam, 1979, theory and numerical analysis, With an appendix by F. Thomasset.

[17] H. Kunita, Stochastic differential equations and stochastic flows of diffeomorphisms, in: École d'été de probabilités de Saint-Flour, XII-1982, Vol. 1097 of Lecture Notes in Math., Springer, Berlin, 1984, pp. 143-303. 